University of New Hampshire

University of New Hampshire Scholars' Repository

Physics Scholarship

Physics

6-1-1991

\title{
ULF waves in the low-latitude boundary layer and their relationship to magnetospheric pulsations: A multisatellite observation
}

\author{
K. Takahashi \\ D. G. Sibeck \\ P. T. Newell \\ Harlan E. Spence \\ Boston University, harlan.spence@unh.edu
}

Follow this and additional works at: https://scholars.unh.edu/physics_facpub

Part of the Physics Commons

\section{Recommended Citation \\ Takahashi, K., D. G. Sibeck, P. T. Newell, and H. E. Spence (1991), ULF waves in the low-latitude boundary layer and their relationship to magnetospheric pulsations: A multisatellite observation, J. Geophys. Res., 96(A6), 9503-9519, doi:10.1029/91JA00612.}

This Article is brought to you for free and open access by the Physics at University of New Hampshire Scholars' Repository. It has been accepted for inclusion in Physics Scholarship by an authorized administrator of University of New Hampshire Scholars' Repository. For more information, please contact Scholarly.Communication@unh.edu. 


\title{
ULF Waves in the Low-Latitude Boundary Layer and Their Relationship to Magnetospheric Pulsations: A Multisatellite Observation
}

\author{
Kazue Takahashi, David G. Sibeck, and Patrick T. Newell
}

The Johns Hopkins University Applied Physics Laboratory, Laurel, Maryland

\section{HARLAN E. SPENCE}

Space Sciences Laboratory, The Aerospace Corporation, El Segundo, California

\begin{abstract}
On April 30 (day 120), 1985, the magnetosphere was compressed at 0923 UT and the subsolar magnetopause remained near $7 R_{E}$ geocentric for $\sim 2$ hours, during which the four spacecraft Spacecraft Changing At High Altitude (SCATHA), GOES 5, GOES 6, and Active Magnetospheric Particle Tracer Explorers (AMPTE) CCE were all in the magnetosphere on the moming side. SCATHA was in the low-latitude boundary layer (LLBL) in the second balf of this period. The interplanetary magnetic field was inferred to be northward from the characteristics of precipitating particle fluxes as observed by the low-altitude satellite Defense Meteorological Satellite Program (DMSP) F7 and also from absence of substorms. We used magnetic field and particle data from this unique interval to study ULF waves in the LL.BL and their relationship to magnetic pulsations in the magnetosphere. The LLBL was identified from the properties of particles, including bidirectional field-aligned electron beams at $\sim 200 \mathrm{eV}$. In the boundary layer the magnetic field exhibited both a 5-10 min irregular compressional oscillation and a broadband $(\Delta f f-1)$ primarily transverse oscillations with a mean period of $\sim 50 \mathrm{~s}$ and a left-hand sense of polarization about the mean field. The former can be observed by other satellites and is likely due to pressure variations in the solar wind, while the latter is likely due to a Kelvin-Helmholtz (K.-H.) instability ocourring in the LLBL or on the magnetopanse. Also, a strongly transverse 3-s oscillation was observed in the LIBL. The magnetospheric pulsations, which exhibited position dependent frequencies, may be explained in terms of field line resonance with a broadband source wave, that is, either the pressureinduced compressional wave or the K.-H. wave generated in or near the boundary layer.
\end{abstract}

\section{INTRODUCTION}

The low-latitude boundary layer (LLBL) is a region of great importance with regard to energy, mass, and momentum transfer from the solar wind into the magnetosphere. Plasma waves in various frequency regimes no doubt play an important role in the transfer processes. In this paper we study the nature of ULF waves (frequency less than the local proton cyclotron frequency, $f_{\mathrm{CH}^{+}}$) observed in this region and the relationship between the waves in LLBL and magnetic pulsations in the magnetosphere. Although we will describe waves with periods shorter than $10 \mathrm{~s}$, our primary interest is in those in the Pc 3-5 band (period $=10-600 \mathrm{~s}$ ).

The presence of ULF waves in the vicinity of the magnetopause is well established. The magnetopause normal is constantly distorted [Fainfield, 1976], and the distortions are consistent with tailward propagating disturbances [Aubry et al., 1971; Ledley, 1971]. Also, ULF plasma and field oscillations are present in the LLBL, as observed in situ [Sckopke et al., 1981; Couzens et al., 1985] and at low-altitude [Potemra et al., 1988]. Often these ULF oscillations are attributed to the Kelvin-Helmholtz (K.-H.) instability [Ogilvie and Fitzenreiter, 1989]. However, Sibeck et al. [1990] reinterpreted oscillations reported by Sckopke et al. [1981] as evidence for K.-H. wave in terms of multiple magnetopause crossings caused by a quasi-periodic variation in the solar wind dynamic pressure. Therefore one purpose of the present study is to distinguish waves generated within the LLBL and those due to extemal pressure perturbations.

One consequence of waves generated near or transmitted from the magnetopause is their coupling with standing Alfven waves in

\section{Copyright 1991 by the American Geophysical Union.}

Paper number 91JA00612.

0148-0227/91/91JA-00612\$05.00 the magnetosphere [Chen and Hasegawa, 1974; Southwood, 1974]. Although smaller than energy transport associated with substorms by 2 orders of magnitude, appreciable energy (as much as $2 \times 10^{20}$ ergs $/ \mathrm{hr}$ ) can be transferred into the magnetosphere via the Alfven waves [Greenwald and Walker, 1980]. Although the idea of the coupling has been generally accepted, no detailed observations have been made simultaneously in the source region (magnetopause or LLBL) and in the resonance region (magnetosphere). Chen and Hasegawa [1974] and Southwood [1974] postulated a monochromatic source wave at the magnetopause, but spacecraft observations very often indicated that multiharmonic toroidal resonances occur in the dayside magnetosphere [Takahashi and McPherron, 1982; Engebretson et al., 1986; Anderson et al., 1990a], and multisatellite observations further established that toroidal resonances occur simulateously at positiondependent frequencies [Takahashi and McPherron, 1984a]. These observations suggest a broadband energy source for magnetospheric pulsations. Therefore another purpose of the present study is to find out the relationship between Pc 3-5 waves occurring in the vicinity of the magnetopause and those occurring in the magnetosphere.

The time interval studied is 0900-1200 UT on day 120 (April 30) of 1985 . On this day the magnetosphere was greatly compressed for $\sim 2$ hours starting at 0923 UT. This made it possible for Spacecraft Charging At High Altitude (SCATHA) to observe the magnetopause proper near its apogee $\left(7.8 R_{E}\right)$ for an extended period ( 1 hour) near 0800 local time. Combined magnetic field and particle data from SCATHA made it possible to determine the nature of ULF waves in the LLBL. Three other spacecraft, Active Magnetospheric Particle Tracer Explorers (AMPTE) CCE, GOES 5, and GOES 6, observed enhanced pulsation activity in the dawn sector of the magnetosphere.

The organization of the paper is as follows. In section 2 the spacecraft and their instrumentation are briefly described. In sec- 
tion 3 we present observations with SCATHA near the magnetopause, including the particle and magnetic field signature of the LLBL and ULF waves in that region. In section 4 we describe magnetic pulsations in the magnetosphere. In section 5 we discuss the generation mechanisms of ULF waves in the LLBL and the coupling of the waves to the magnetospheric pulsations.

\section{INSTRUMENTATION}

We mainly use magnetic field and particle data acquired by four high-altitude spacecraft, SCATHA (also known as P78-2), AMPTE CCE, GOES 5, and GOES 6. SCATHA has an apogee of 7.8 $R_{E}$, a perigee of $5.3 R_{E}$, an inclination of $7.8^{\circ}$, and an orbital period of 23.6 hours. The satellite is spin stabilized with a spin axis parallel to the spacecraft orbital plane and nearly perpendicular to the Sun-Earth line. It had a spin period of $62 \mathrm{~s}$ for the time interval studied. CCE has an apogee of $8.8 R_{E}$, a perigee of $1.2 R_{E}$, an inclination of $4.8^{\circ}$, and an orbital period of 15.7 hours. $C C E$ is also spin stabilized with a spin axis maintained within $30^{\circ}$ of the SunEarth line and a spin period of $5.9 \mathrm{~s}$. The GOES spacecraft are geostationary at geographic longitudes of $74^{\circ} \mathrm{W}$ (GOES 5) and $108^{\circ} \mathrm{W}$ (GOES 6).

All these spacecraft are provided with flux gate magnetometers. Each magnetometer has an amplitude resolution equal to or better than $0.2 \mathrm{nT}$, which is sufficient for studying ULF waves near the magnetopause and in the magnetosphere. The time resolution is $0.25 \mathrm{~s}$ for SCATHA, $0.124 \mathrm{~s}$ for CCE, and $3 \mathrm{~s}$ for GOES 5 and GOES 6. Descriptions of the experiments are given by Fennell [1982] for SCATHA, by Potemra et al. [1985] for CCE, and by Grubb [1975] for GOES 5 and GOES 6.

Particle data useful for the present study are available from SCATHA and CCE. These are acquired with the low-energy electron and ion experiment, called SC2, on SCATHA [Fennell, 1982] and with the medium-energy particle experiment (MEPA) on CCE [McEntire et al., 1985]. Both instruments use spacecraft spin to measure the pitch angle distributions. SC2 covers an energy range of $6 \mathrm{eV}$ to $18.6 \mathrm{keV}$ for electrons and $5 \mathrm{eV} /$ charge to 15.6 $\mathrm{keV} /$ charge for ions. As for MEPA, we use data from the front microchannel plate (MCP1) of the time-of-flight head and from the ion head (ECHn, where $n$ designates the channel number). MCP1 data are a crude measure of the relative flux intensity of ions with energy greater than 4-10 keV, and ECHn give a measure of the flux of ions at energies greater than $25 \mathrm{keV}$. In addition, we use precipitation particle fluxes measured by the low-altitude $(\sim 835 \mathrm{~km})$ polar-orbiting satellite Defense Meteorological Satellite Program (DMSP) F7 [Hardy et al., 1984]. The DMSP data are used to infer the direction of the interplanetary magnetic field (IMF).

\section{OBSERVATIONS}

\subsection{Geomagnetic Background}

During the time interval studied the magnetosphere was strongly compressed, apparently because of enhanced solar wind dynamic pressure. Figure 1 shows the geomagnetic indices Dst and $K p$ during this interval, and Figure 2 shows the ordinary magnetogram from Fredericksburg (invariant latitude $=50.11^{\circ}$, magnetic longitude $=356.34^{\circ}$, local time $\sim$ UT -5 hours) along with the $A E$ index. The $H$ component at Fredericksburg suddenly increased at 0923 UT ( $\Delta H \sim 30 \mathrm{nT})$ and remained at an elevated level until 1140 UT. The $H$ increase has been classified as a storm sudden commencement (SSC), so the magnitude of the field change can be explained by the change of the size of the magnetopause, i.e., the change in the Champan-Ferraro current [Mead, 1964]. For example, a displacement of the subsolar magnetopause from $10 R_{E}$ to 7

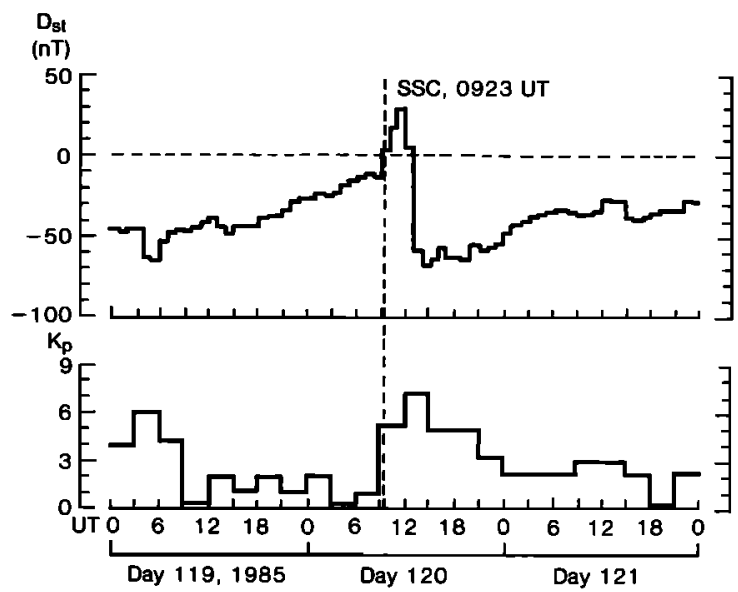

Fig. 1. Geomagnetic indices (top) Dst and (bottom) $K p$ at the time of the storm sudden commencement on April 30 (day 120), 1985.

$R_{E}$ accounts for the observed $\Delta H$ at Predericksburg. Except for the spike at $0923 \mathrm{UT}, A E$ remained below $150 \mathrm{nT}$ between 0923 and 1140 UT, which suggests that energy input from the solar wind to the magnetosphere by reconnection was small. At $1140 \mathrm{UT}, A E$ began to increase and reached a maximum of $\sim 2000 \mathrm{nT}$ at 1300 UT. This clearly indicates a substorm activity. Similarly, $H$ at Fredericksburg started to decrease at $1140 \mathrm{UT}$, accompanied by an irregular oscillation, and reached a minimum around 1320 UT. This $\boldsymbol{H}$ decrease below previous steady values is the result of the

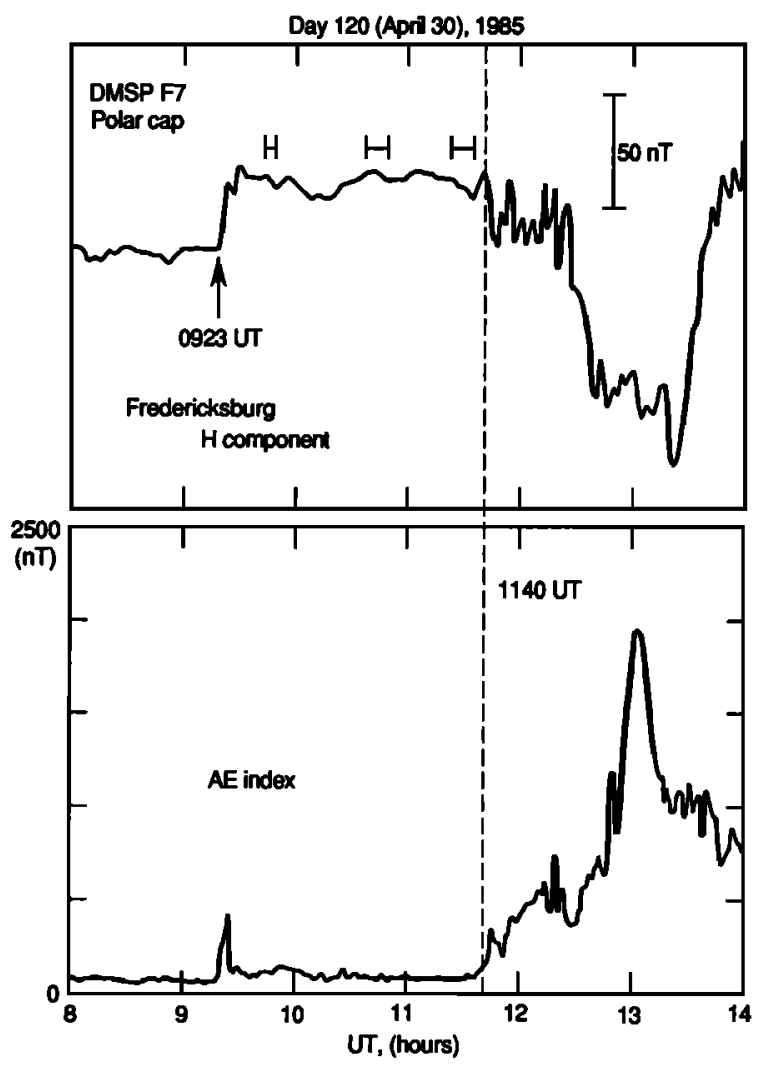

Fig. 2. Ground magnetogram from Fredericksburg and the $A E$ index for the storm sudden commencement on day 120,1986. Local time of Fredericksburg is universal time minus $\sim 5$ hours. The horizontal bars indicate the time intervals during which the DMSP F7 satellite was in the polar cap. 


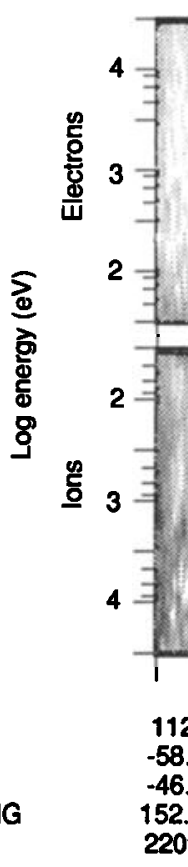

April 30 (day 120), 1985

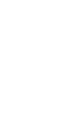

UT
MLAT
GLAT
GLONG
MLT

DMSP F7

April 30 (day 120), 1985

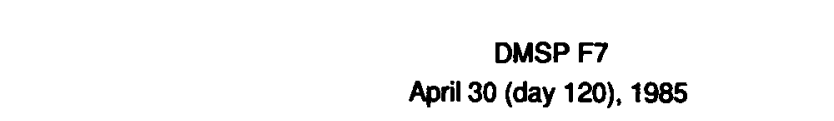

$\log f(u x$
$\left(\mathrm{eV}\left(\mathrm{cm}^{2} . \mathrm{s.sr} \cdot \mathrm{eV}\right)^{-1}\right)$

$\mathrm{V}\left(\mathrm{cm}^{2} . \text { s.sr.eV }\right)^{-1}$

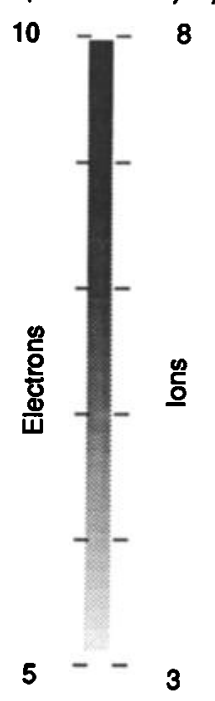

Fig. 3. DMSP F7 precipitation particle data suggesting northward IMF.

development of a stomm time ring current, which is consistent with the large dip in the Dst index which is evident after 1200 UT (Dst has a time resolution of 1 hour). We attibute the enhanced geomagnetic activity after 1140 UT to southward orientation of the IMF, as will be dicussed below.

During the period from 0930 to 1140 UT the IMP was probably oriented northward (unfortunately, there were no in situ solar wind plasma or IMF observation). The evidence comes from the lowaltitude satellite DMSP F7. During its polar cap passes at 0943-0952 UT, 1037-1048 UT, and 1123-1133 UT its particle detector found electron precipitation patterns characteristic of a northward IMF. Figure 3 is a spectrogram showing a sample DMSP F7 polar pass, from 1123 to 1133 UT. Instead of a smooth polar rain or empty polar cap (which would be indicative of southward IMF $B_{z}$ ), the polar cap is filled with bursty highly structured precipitation, which is characteristic of northward $B_{2}$ [e.g., Hardy et al., 1986; Lassen et al., 1988]. Polar cap arcs, well known to be a northward $B_{2}$ phenomenon are present at $1129: 30$ ( $-89.4^{\circ}$ MLAT; $\left.10 \mathrm{ergs} / \mathrm{cm}^{2} \mathrm{~s}\right)$ and at $1132: 11\left(-81.5^{\circ} \mathrm{MLAT}, 50 \mathrm{ergs} / \mathrm{cm}^{2} \mathrm{~s}\right)$. Other evidence that $B_{z}$ is probably northward comes from the lack of clear or strong discrete auroral arcs during the transition through the nightside (2210 MLT) portion of the auroral oval. One important consequence of the northward IMF is that there was no substorm activity until 2 hours after the compression of the magnetosphere, as will be shown in section 3.3. After 1140 UT, SCATHA was in the magnetosheath and observed predominantly southward sheath field.

The magnetospheric compression made it possible to observe the outer magnetosphere and the LLBL with spacecraft that usually stay in the midmagnetosphere (i.e., at or near geostationary orbit). In addition, it was fortunate that the compression did not cause magnetospheric substorms immediately. Substorms would have generated internal plasma and field disturbances which would have obscured ULF waves of extemal origin.
The orbit segments of the spacecraft for 0930-1130 UT are shown in Figure 4. The spacecraft were all located on the moming side at northem dipole latitudes. SCATHA was the closest to the magnetopause and made multiple magnetopause crossings starting at 1140 UT at a radial distance of $7.5 R_{E}$, at a magnetic local time of -0830 , and at a dipole latitude of $14^{\circ}$. Included in the figure is the average magnetopause cross section from Fairfield [1971] and its compressed version which gives an approximate magnetopause location for 0930-1130 UT. Although we do not have solar wind dynamic pressure data to find the exact location of the magnetopause, there is evidence from particle and magnetic field measurements that SCATHA was very close to the magnetopause at least between 1040 and 1140 UT.

\subsection{SCATHA Observations Near the Magnetopause}

The prolonged interval of the magnetospheric compression on day 120 , provided us with a rare opportunity of observing the

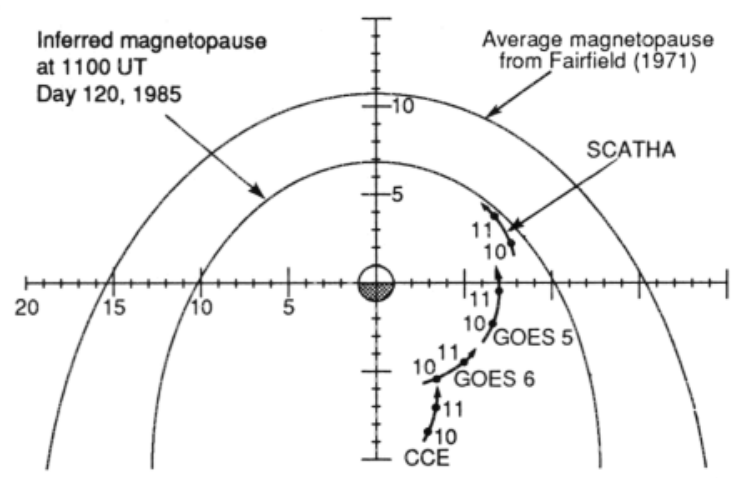

Fig. 4. Local time versus $L$ plots of the orbit segments of four spacecraft for the time interval of 0930 to 1130 UT on April 30, 1986. Two magnetopause positions are illustrated to indicate the magnitude of the magnetospheric compression on day 120. 
SCATHA, DAY 120, 1985

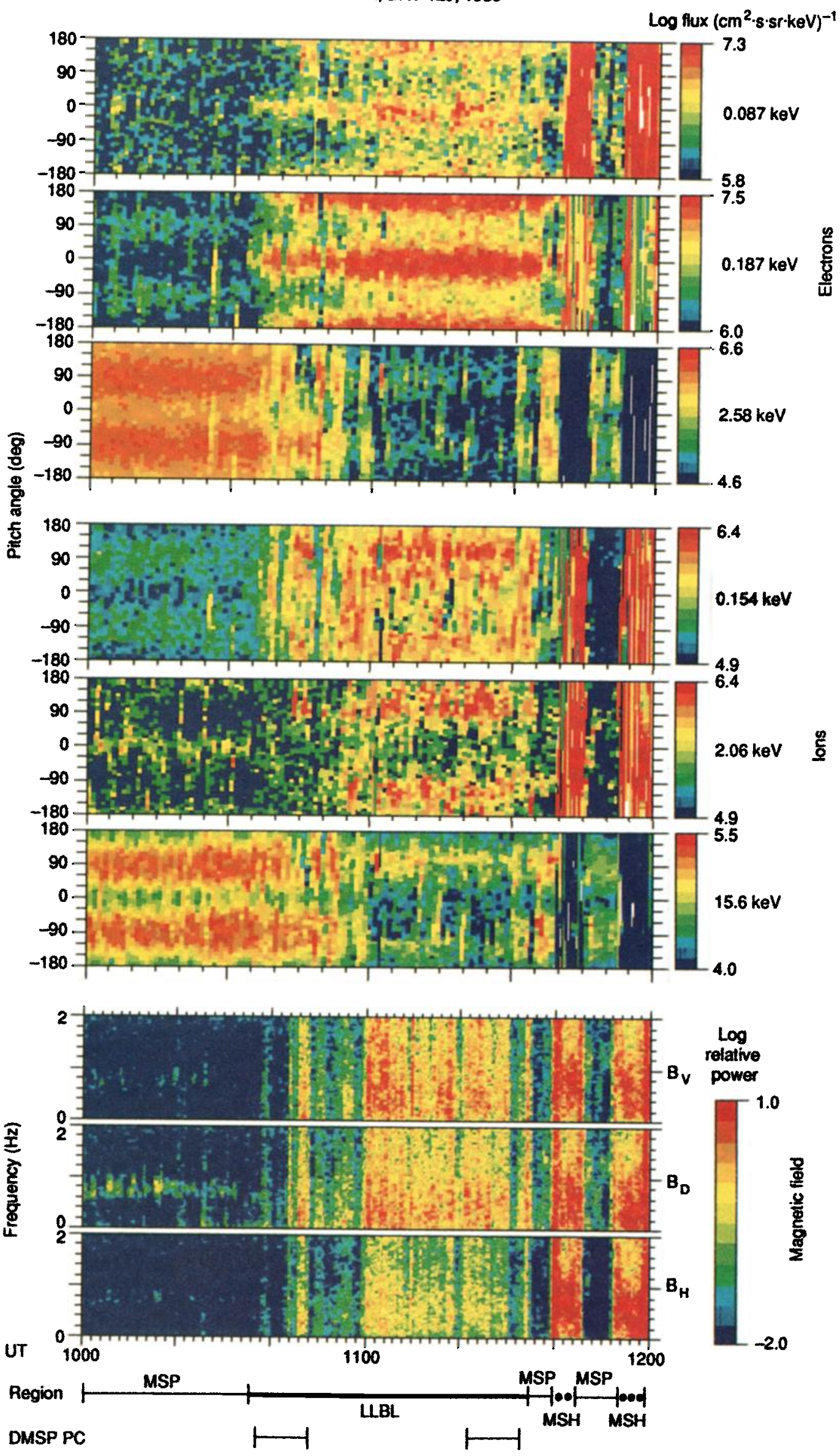


LLBL on the moming side with SCATHA. We first present particle and magnetic field data from SCATHA in order to identify different regions near the magnetopause and then describe the properties of magnetic field oscillations in these regions.

Particle and magnetic field regimes. The upper six panels of Plate 1 show differential flux from selected channels of the SC2 instrument, as a function of time and pitch angle. The vertical axis gives the pitch angle and has a range of $-180^{\circ}$ to $180^{\circ}$ with the positive (negative) angles corresponding to the sunward (antisunward) look directions of the detector. The instrument does not always measure precisely field-aligned fluxes $\left(0^{\circ}\right.$ or $180^{\circ}$ pitch angles) during a satellite spin. Accordingly, the pixels for fieldaligned directions are filled with data from the most field-aligned measurements. For the magnetospheric interval (including LLBL) the minimum angle between the look direction of the particle detector and the ambient magnetic field was less than $20^{\circ}$ (see Figure 9 for the pitch angle coverage in the LLBL).

Three particle regimes can be identified. These are the magnetosheath (MSH in the region identifier shown at the bottom of the figure), the LLBL, and the magnetosphere (MSP). The magnetosheath manifests itself by disordered pitch angle distributions owing to the variability of the magnetic field direction. There are strong fluxes of 0.087-keV electrons and 0.154- and 2.06-keV ions. They represent the shocked solar wind plasma. At a higher energy (15.6 keV), ion flux is considerably lower than in the magnetosphere or in the boundary layer. Apparently, the magnetospheric ions at this energy either did not escape into the magnetosheath with a high flux intensity or did not reach the spacecraft.

The particle signatures in the low-latitude boundary layer and the magnetosphere differ strikingly. First, the overall intensity of the flux in a given channel changes. In the $0.087-\mathrm{keV}$ electron channel the flux increases from the magnetosphere to the boundary layer and further to the magnetosheath. A similar change is seen for ions at 0.154 and $2.06 \mathrm{keV}$. At the highest energies (2.5-keV electrons and 15.6-keV ions) the flux is greatest in the magnetosphere and decreases upon entry into the magnetosheath. These results qualitatively agree with previous observations that the energy spectrum in the boundary layer is intermediate between the magnetosphere where high-energy particles dominate and the magnetosheath where low-energy particles dominate [Eastman et al., 1976; Bryant and Riggs, 1989]. Second, the pitch angle distribution shows a characteristic change from one region to another. In the magnetosphere, both electrons and ions have maximum flux at $90^{\circ}$ pitch angle except for the $0.154-\mathrm{keV}$ ions. In the boundary layer, electrons have a bidirectional field-aligned distribution at 0.087 and $0.187 \mathrm{keV}$, while at $2.58 \mathrm{keV}$ the pitch angle distribution appears to have a peak at $90^{\circ}$. Inspection of other electron channels indicates that a field-aligned distribution is present up to $\mathbf{0 . 8 1 7}$ keV.

The electron distribution forms well-defined counter streaming beams along the ambient field. Figure 5 shows the average phase space density of electrons for the time interval of 1123-1128 UT. The beams are evident as the strong peaks at $8000 \mathrm{~km} / \mathrm{s}(0.187$ $\mathrm{keV}$ ) in the parallel distribution, $f_{\mathrm{f}}$. The parallel distribution is greater than the perpendicular distribution, $f_{\perp}$, except at the highest

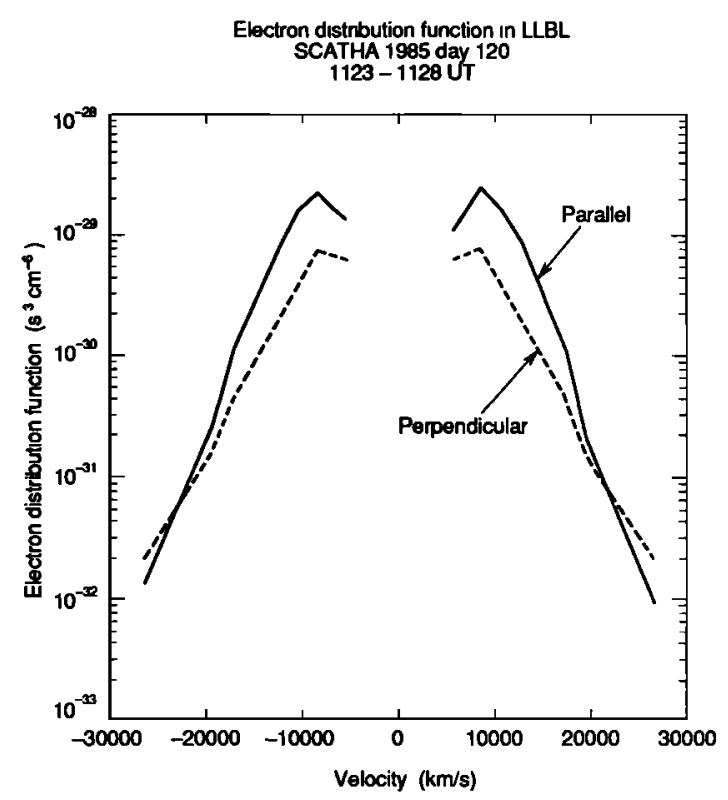

Fig. 5. Parallel and perpendicular cuts of electron distributions from SCATHA for a 5-min interval in the LLBL. For the parallel velocity the positive (negative) sign means electron motion parallel (antiparallel) to the magnetic field. For the perpendicular velocity the sign has no meaning (the distribution is symmetric about 0 velocity).

velocity, $27000 \mathrm{~km} / \mathrm{s}(1.94 \mathrm{keV})$. At $8000 \mathrm{~km} / \mathrm{s}$ the ratio $f_{i l} f_{\perp}$ is approximately 3 . A peak also occurs in $f_{\perp}$ at the same velocity, which implies that either the electrons were accelerated both parallel and perpendicular to the magnetic field or they were pitch angle scattered after being accelerated in one direction.

Electrons with a similar bidirectional distribution have been observed previously. Ogilvie et al. [1984] found electron beams at

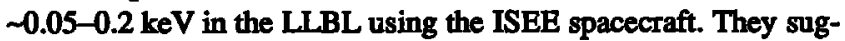
gested that the distribution has the same origin as the bidirectional electron distributions observed at low altitudes [Sharp et al., 1980; Collin et al., 1982; Klumpar and Heikkilla, 1982; Burch et al., 1983]. Another bidirectional electron distribution was observed by Farrugia et al. [1988] in association with magnetic field/particle variations that were interpreted as flux transfer events (FTE). In the FTE model the field-aligned beams result from merging and ionospheric mirroring. However, the same events were reinterpreted by Sibeck [1990] in terms of boundary motion, and the region of the bidirectional electron streaming was identified to be the LLBL. Since we believe the IMF to have been northward at the time of the LLBL observations presented in this paper, the bidirectional distributions may be taken as evidence that LLBL (as defined in the present paper) is connected to the ionosphere, rather than to the solar wind.

Bidirectional electron distributions are seen adjacent to the magnetopause crossings at 1140 and 1145 UT, indicating that LLBL was present during the radial motion of the magnetopause. For these cases the distributions were observed only in one spin (62 s) of the satellite, implying either that the radial motion of the magne-

Plate 1. (Opposite) Color-coded differential fluxes of particles and dynamic power spectra from SCATHA for 1000-1200 UT including a prolonged interval in the low-latitude boundary layer (LLBL). Three regions are identified from the data as indicated at the bottom: the magnetosphere (MSP), the LIBL, and the magnetosheath (MSH). Polar cap passes by the DMSP F7 satellite are also indicated (DMSP PC). The pitch angle distribution in the magnetosheath should not be trusted becanse the software for the time-pitch angle display assumes a monotonically changing pitch angle during a satellite spin, which is not always the case in the magnetosheath. 
topause was fast (these LLBL intervals are not shown in the region identifier of Plate 1) or that the width of the region in which bidirectional distribution occurred had decreased. We are not able to identify the boundary layer signature for the magnetopause crossing at $1152 \mathrm{UT}$.

The transition from the magnetosphere to the LLBL is not necessarily abrupt, and the timing of spacecraft entry into the LLBL depends on particle species. For example, the $0.187-\mathrm{keV}$ electrons change from a low-flux and pancake distribution to a high-flux and field-aligned distribution at 1035 UT. However, in the $15.6-\mathrm{keV}$ ion data the flux intensity remained magnetospherelike until 1054 UT. This is not unexpected, because the ions have a larger gyroradius $(-100 \mathrm{~km}$ at $15.6 \mathrm{keV})$ than the electrons $(0.2 \mathrm{~km}$ at 0.087 $\mathrm{keV}$ ) and the transition from the magnetosphere to the LLBL could occur over a distance comparable to the ion gyroradius. In Plate 1 we have defined the boundary layer in terms of $0.087-$ and 0.187-keV electrons.

Magnetic field data for the 1000-1200 UT interval are shown in Figure 6. The plots were made from 5-s averaged field components $B_{W} B_{D}, B_{H}$, and the magnitude $B_{T}$ where $\hat{e}_{H}$ (northward) is parallel to the Earth's spin axis, $\hat{e}_{D}$ is geographically eastward, and $\boldsymbol{e}_{V}=\hat{e}_{D} \times \hat{\epsilon}_{H}$. The radial component, $\hat{e}_{V}$, would differ from the unperturbed local magnetopause normal by $\sim 20^{\circ}$. In the magnetosphere, $B_{H}$ is nearly equal to $B_{T}$.

We describe the magnetic fields with reference to the region identifier from the particle data, shown between the traces for $B_{H}$ and $B_{T}$. The magnetosheath is evident from its southward magnetic field orientation which differs considerably from that in the magnetosphere. In the boundary layer the field magnitude is somewhat elevated from the magnetospheric level. This can be attributed to the gradual compression of the magnetosphere that occurred from $\sim 1020$ UT. The compression was such that SCATHA stayed in the

SCATHA magnetometer Aprll 30 (day 120), 1985

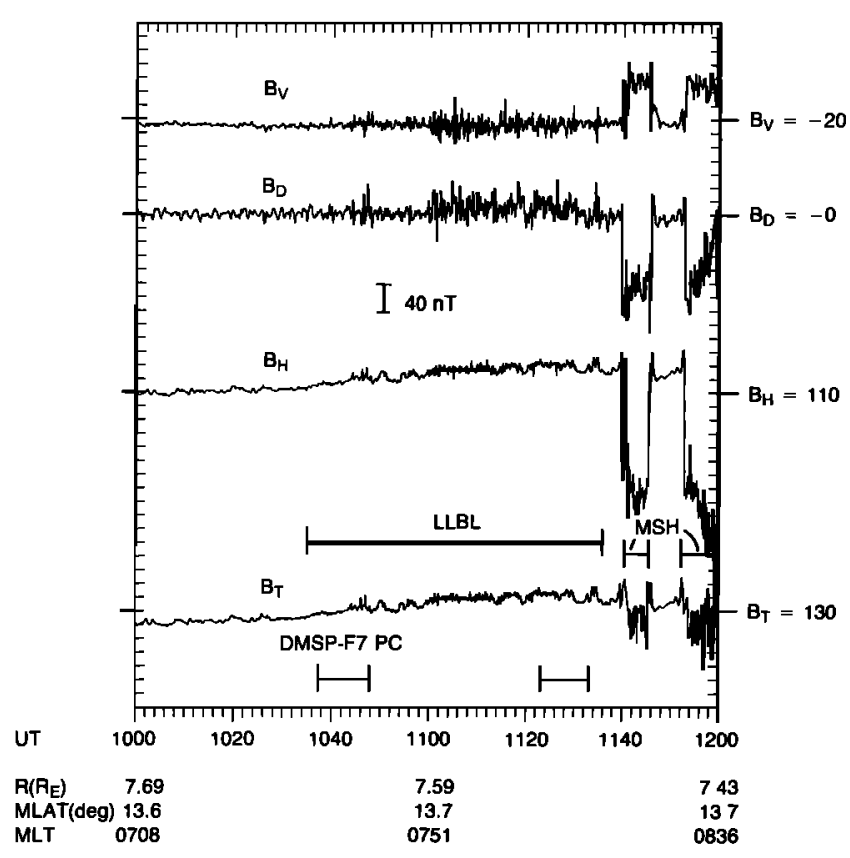

Fig. 6. Magnetic field data showing SCATHA's approach to and crossings of the magnetopause. The data are 5-s averages calculated from 0.25-s data. Region identification from polar cap passes of the DMSP F7 satellite are also shown.
LLBL as the satellite moved earthward. We also note that when there is a large-amplitude oscillation in the boundary layer, the mean value of $B_{D}$ is slightly larger. For example, the time average of $B_{D}$ for $1050-1100 \mathrm{UT}$ (magnetosphere) is $\sim 2 \mathrm{nT}$ as compared to $\sim 14 \mathrm{nT}$ for 1100-1115 UT (LLBL). This implies that a fieldaligned current flows at or near the magnetosphere/LLBL interface. The $\sim 12 \mathrm{nT}$ difference is equivalent to a planer current with an intensity of $1.0 \times 10^{-2} \mathrm{~A} / \mathrm{m}$. Furthermore, the larger $B_{D}$ in the boundary layer implies a northward current, i.e., from the equator toward the ionosphere. Thus the current flows in the same direction as the region 1 current of Iijima and Potemra [1976]. A connection between Region 1 currents and the LLBL has previously been suggested by Eastman et al. [1976], and Hones [1983] used ISEE 1 and 2 magnetic field observations in systematic search for the current signature. Hones found that the field in the boundary layer was very often tilted in the direction opposite to that expected from "enhanced draping," a configuration in which the LLBL field lines are pulled most tailward at the equator rather than at a higher latitude (see Figure 2 of Hones' paper). Our case corresponds to enhanced draping, suggesting that boundary layer magnetic field was dragged tailward by a force operating at the equator. The difference between the present study and Hones [1983] may be due to the lower latitude of SCATHA. Another possibility for the $12 \mathrm{nT}$ difference in $B_{D}$ is that the solar wind condition changed at $\sim 1100$ UT, bringing the LLBL to the location of SCATHA and also changing the orientation of the magnetic field in the LLBL. Unfortunately, we cannot test this possibility because of a lack of observations of the IMF and solar wind plasma at $~ 1100 \mathrm{UT}$.

There are considerable field oscillations in the boundary layer in the Pc 3-5 band (periods longer than $10 \mathrm{~s}$ ). First, $B_{T}$ shows an irregular oscillation with peaks separated by $5-10 \mathrm{~min}$ and peak-topeak amplitude of $\sim 20 \mathrm{nT}$ or less. This oscillation is not limited to the LLBL but is also observed in the magnetospheric portion of the data without much reduction in amplitude. Second, a large-amplitude ( $-50-\mathrm{nT}$ peak-to-peak) oscillation is evident in the radial $\left(B_{V}\right)$ and azimuthal $\left(B_{D}\right)$ components. The compressional component oscillates also, but its amplitude is much less than that of the transverse components. In the magnetosphere there is an oscillation in $B_{D}$ with amplitude less than $20 \mathrm{nT}$ peak to peak. Much of the oscillation, however, is likely the result of satellite nutation at 1-2 $\mathrm{min}$ period (J. F. Fennell, personal communication, 1989).

The lower three panels of Plate 1 illustrate higher-frequency oscillations for 1000-1200 UT using color-coded Fast Fourier transform (FFT) dynamic spectra from the full time resolution $(0.25 \mathrm{~s})$ magnetic field data. The spectra were calculated using 200 data points (50 s) with $50 \%$ overlap in successive steps. The color code indicates the power spectral density relative to a reference power law spectrum, $\left(f f_{0}\right)^{-2}$ with $f_{0}=1 \mathrm{~Hz}$. The local proton gyrofrequency $(1.8-2.0 \mathrm{~Hz})$ is near the upper frequency limit of display except in the magnetosheath. As was the case with the particle data, the magnetosheath is the easiest to identify: it exhibits a strong broadband spectrum in all components. The magnetosphere is characterized by a quiet background and a discrete oscillation in $B_{D}$ near $0.8 \mathrm{~Hz}\left(\sim 0.4 f_{c \mathrm{ct}^{r}}\right)$. Elliptically polarized ioncyclotron waves near $0.4 f_{c \mathrm{H}^{+}}$have often been observed by $\mathrm{CCE}$ in the dayside magnetosphere near apogee of $\left(8.8 R_{E}\right)$ of the spacecraft [Anderson et al., 1990 b] and our 0.8-Hz wave appears to be of the same type. The transition from the magnetosphere to the LLBL, as determined to be at 1035 UT from the electron observations, is not abrupt. Starting at 1035 UT, wave power in all components increases gradually, reaching a maximum at 1046 UT. The power goes down somewhat (but remains at a level distinctively higher than in the magnetosphere) until 1100 UT, when suddenly it is 
elevated and remains at a high level until 1130 UT. Both in the magnetosphere and in the LLBL, the field oscillations are primarily transverse to the ambient field.

The variation of oscillation amplitude within the LLBL suggests that either the wave amplitude spatially varied within the boundary layer or there was a temporal variation in the solar wind condition that briefly suppressed wave amplitude at $1050-1100$ UT. Conceming the former possibility, we note that Song et al. [1990] suggested in a case study that the LLBL near the subsolar point consists of a inner layer and an outer layer and that the field variation in the $\mathbf{0 . 4 - 2} \mathrm{Hz}$ band is stronger in the outer layer. Our result is qualitatively consistent with the Song et al. observation; hence the two-layer structure of LLBL might be common. To confirm that such a structure is spatial rather than temporal, of course, requires a simultaneous plasma observation in the solar wind. This cannot be done in the present study.

$U L F$ waves near the magnetopause. We now examine in more detail magnetic field oscillations in or near the LLBL. Figure 7 shows examples of high-time-resolution (0.25 s) magnetic field

SCATHA magnetometer April 30 (day 120), 1985
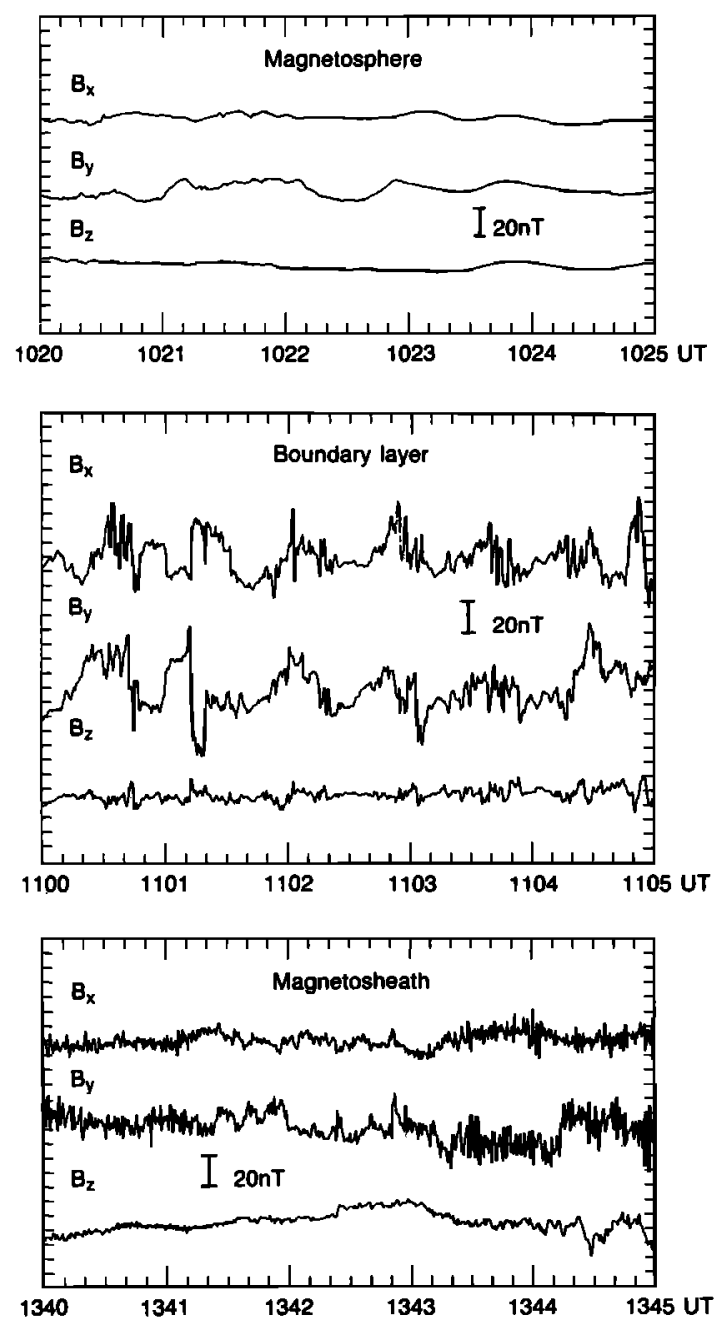

Fig. 7. High-time-resolution $(0.25 \mathrm{~s})$ magnetic field data from different regions near the magnetopause. The field is rotated into local field-aligned coordinates, in which $\ell_{z}$ (parallel component) is along the direction of the average magnetic field for the time interval displayed, $\hat{e}_{y}$ (aximnthal component) is in the direction of $\hat{l}_{z} \mathbf{x}$, where $r$ is the position vector of the spacecraft with respect to the center of the Earth, and $\hat{k}_{x}$ (radial component) $=c_{y} \times \hat{c}_{2}$. data from the three regions. To separate compressional and transverse oscillations, we used a field-aligmed coordinate system, in which $\ell_{z}$ is along the average field for each 5-min interval shown, $\ell_{y}$ is aximuthal, and $z_{x}$ is radial. Figure 8 shows power spectra calculated from the same data. The straight line drawn in each frame of this figure represents an $f^{-2}$ power spectrum which gives a base line for comparing spectra from different components and regions.

The magnetosphere is characterized by the absence of highfrequency oscillations in the time plots. Low-frequency (period $\sim 1$ min) variations are present in $B_{x}$ and $B_{y}$ but they are likely caused by the nutation of the satellite as noted above. We cannot identify naturally excited magnetic pulsations for this interval. However, the field magnitude (nearly equivalent to $B_{z}$ ) is not affected by the nutation. Spectral peaks occur at $0.1 \mathrm{~Hz}$ in $B_{x}$ and $B_{z}$, and at $0.8 \mathrm{~Hz}$ in $B_{y}$. The origin of the $0.1-\mathrm{Hz}$ peak is not known at present. However, it is not a persistent feature in the magnetosphere. The 0.8-Hz peak is likely due to an ion-cyclotron wave as suggested above.

In the boundary layer a quasi-periodic oscillation (average period $\sim \mathbf{5 0} \mathrm{s}$ ) is evident in the transverse components. It has an amplitude of $50 \mathrm{nT}$ peak to peak, which far exceeds the oscillation caused by the satellite nutation. The oscillation is evident as a lowfrequency peak in the spectra at $20 \mathrm{mHz}$ for $B_{x}$ and $B_{y}$ (Figure 8, middle panels). The two transverse components have comparable amplitudes but do not oscillate in phase: $\boldsymbol{B}_{\boldsymbol{y}}$ leads $\boldsymbol{B}_{\boldsymbol{x}}$. This implies a left-hand polarization about the mean field. Although we will use the term 50-s wave hereinafter, it should be noted that the specral width of the wave is quite large. For instance, from the $1100-1120$ UT spectrum which will be shown in Figure 19, we estimate the full width at half maximum, $\Delta f$, to be roughly $20 \mathrm{mHz}$ or $\Delta f f f$ to be near unity. Nevertheless, we have found that $B_{y}$ leads $B_{x}$ even when their waveforms are irregular. Thus the polarization sense is predominantly left-handed in the boundary layer.

Because the 50-s oscillation is transverse, it should be accompanied by a bending of the magnetic field. The 50-nT peak-to-peak amplitude in the 150-nT background is equivalent to an angular perturbation of $20^{\circ}$ peak-to-peak. To find the field line configuration related to the field oscillation, we compare in Figure 9 the magnetic field with electron fluxes at 0.087 and $2.58 \mathrm{keV}$. These energies were chosen as good indicators of the magnetosphere $(2.58 \mathrm{keV})$ and the magnetosheath $(0.087 \mathrm{keV})$. As can be seen in Plate 1, 0.087-keV electrons show a distinctively high flux $\left(-4 \times 10^{7}\left(\mathrm{~cm}^{2} \mathrm{~s} \text { sr keV}\right)^{-1}\right)$ in the magnetosheath, while $2.58-\mathrm{keV}$ electrons show a distinctively high flux $\left(\sim 2 \times 10^{6}\right.$ $\left.\left(\mathrm{cm}^{2} \mathrm{~s} \mathrm{sr} \mathrm{keV}\right)^{-1}\right)$ in the magnetosphere. During the interval of 50-s oscillation, large fluxes of 2.58-keV electrons were observed, notably at 1101:40 1102:50, 1103:20, and 1104:40 UT, all in association with a minimum of $\delta B_{x}$. These enhancements are not a result of the average pitch angle distribution that is peaked at $90^{\circ}$. Because the high flux level is characteristic of the magnetosphere we conclude that the field line displacement that caused the minima of $\delta B_{x}$ brought the magnetospheric plasma to the close vicinity of the satellite. On the other hand, there was no indication in the 0.087-keV electron flux that the spacecraft entered the magnetosheath. The flux variation in this channel was dominated by a bidirectional field-aligned distribution characteristic of the boundary layer, and the flux never reached the high level (indicated by a horizontal dashed line) characteristic of the solar wind.

From these observations we infer the meridional structure of the 50-s oscillation as illustrated in Figure 10. The relative location of the boundary layer with respect to SCATHA is illustrated for two epochs of $\delta B_{x}$. We assume that the field line displacement is sym- 
SCATHA magnetometer April 30 (day 120), 1985
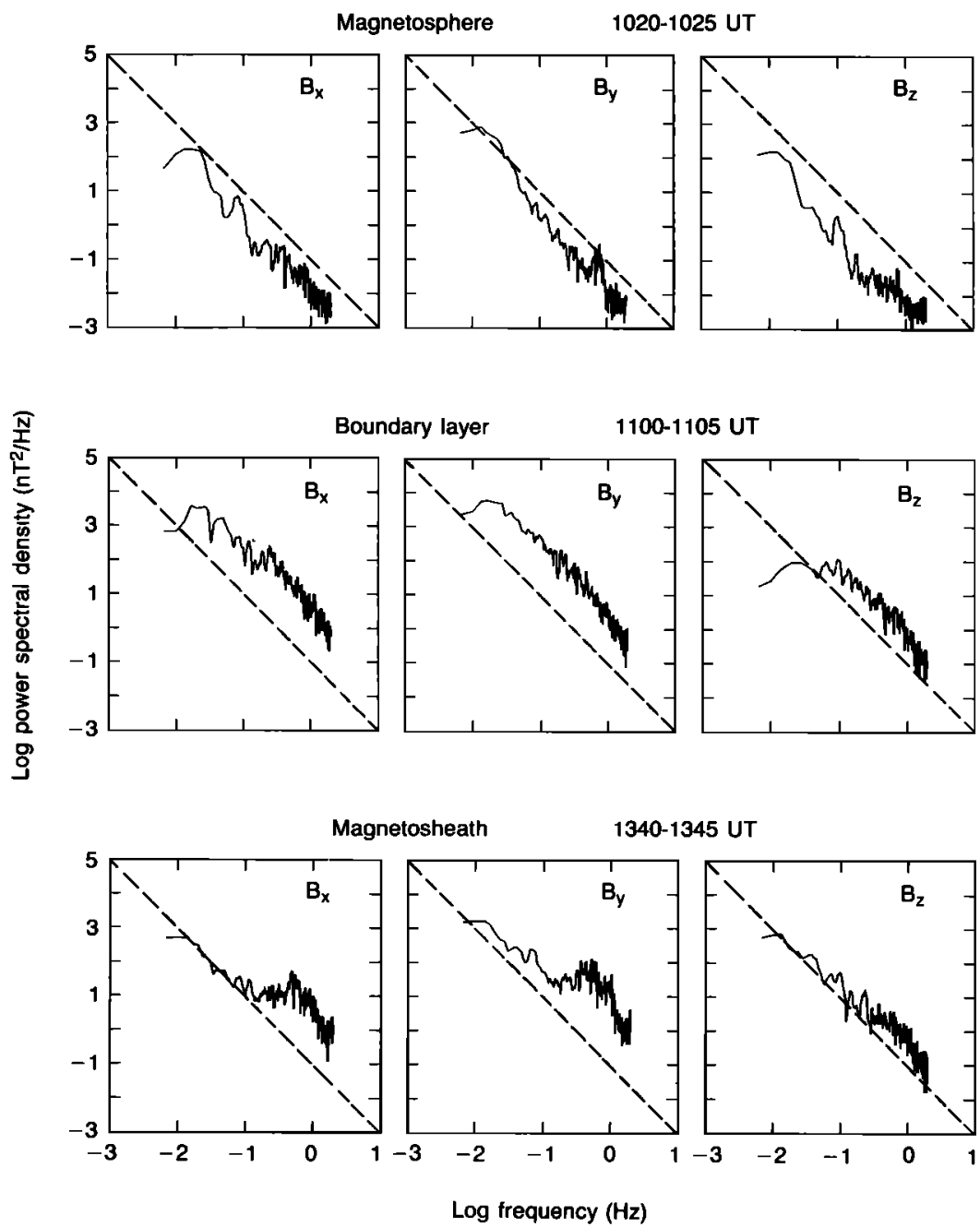

Fig. 8. Power spectra calculated from the magnetic field data shown in Figure 7. The diagonal lines represent a reference power level with a slope of -2 . The local proton gyrofrequency is near the Nyquist frequency $(2 \mathrm{~Hz}$ ) of the magnetic field data.

metric about the magnetic equator, with a characteristic amplitude scale length of $\ell_{\| 1}$. This is similar to the structure of odd-mode standing waves on geomagnetic field lines [Sugiura and Wilson, 1964]. A wave propagating along the field line can be excluded, because for such a wave the magnetospheric plasma would be encountered at the descending or ascending node of the $\delta B_{x}$ oscillation. A standing wave with an antisymmetric structure can also be excluded, because for such a wave the the magnetospheric plasma would be encountered at the maxima of $\delta B_{x}$ at the SCATHA location, provided $l_{1}$ is not too small.

Using Figure 10, we relate $\delta B_{x}$ to the amplitude of radial flux tube motion. We simply assume that the radial field line displacement is given by

$$
\xi_{x}=\xi_{e q} \cos \left(k_{z} z\right)
$$

where $\xi_{a q}$ is the equatorial displacement, $k_{z}=2 \pi / \ell_{\| 1}$, and $z$ is distance from the equator. Then, further neglecting the field in- homogeneity and curvature and assuming $\left|k_{z} z\right| \ll 1$, the observed field perturbation is related to $\xi_{\text {eq }}$ as

$$
\xi_{e q}=\delta B_{x}\left(B k_{z}^{2} z\right)^{-1}
$$

With the observed values of $B=150 \mathrm{nT}$ and $\delta B_{x}=25 \mathrm{nT}$ (half of the peak-to-peak amplitude) at $z \sim 1.8 R_{E}$, and with an assumed value of $\ell_{\| 1}=10 R_{E}$, we get $\xi_{e q}=0.5 R_{E}$ peak to peak. If we assume that the thickness of the boundary layer did not change, then this displacement and the fact that SCATHA did not enter the magnetosheath imply that the thickness of the boundary was greater than $0.5 R_{E}(-3000 \mathrm{~km})$. Note that this estimate could be in gross error if the value of $\ell_{\|}$was wrong or if the thickness of the boundary layer changed during the oscillation as has been suggested by Sckopke et al. [1981].

In addition to the 50-s oscillation there is another type of oscillation with a period of a few seconds or shorter. An example of a $1-\min$ interval including such a wave is shown in Figure 11. The 

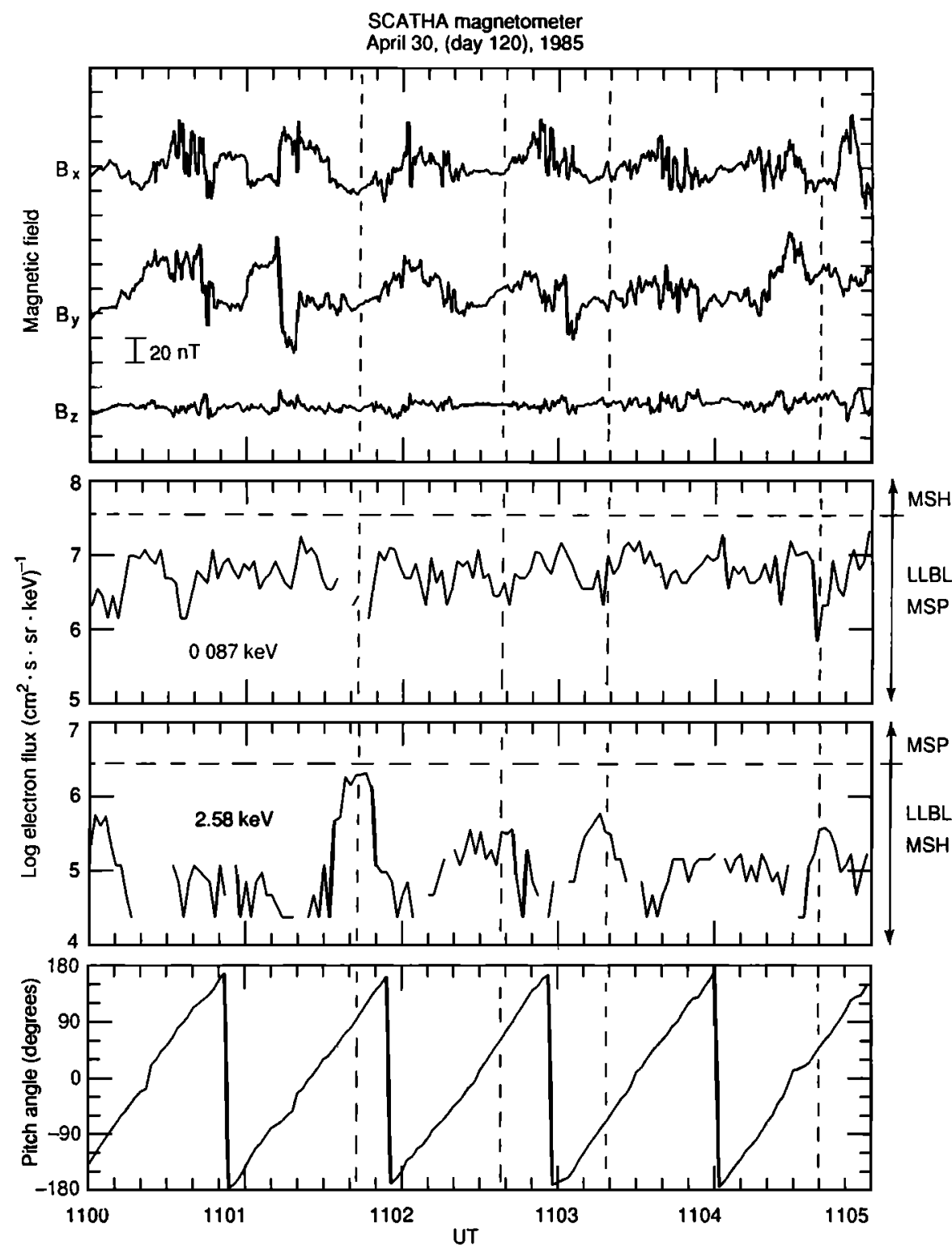

Fig. 9. Relation between magnetic field and electron fluxes during an interval of a 50-s oscillation in the LLBL. Vertical dashed lines indicate minima of $\boldsymbol{B}_{\boldsymbol{x}}$.

oscillation is again primarily transverse with an amplitude of 10-20 nT peak to peak. Although the oscillation is not really monochromatic, it still has a weak spectral peak at $\sim 0.3 \mathrm{~Hz}$ in the power spectrum for $B_{x}$ (Figure 8). To simplify notation, we call it a 3-s oscillation. As is already clear from Figure 9, the amplitude of the 3-s oscillation is modulated by the 50-s oscillation. To emphasize this point we illustrate in Figure 12 low- and high-pass-filtered versions of the radial magnetic field component. The amplitude of the 3-s oscillation is largest at the maxima of the 50-s oscillation. In terms of the field line motion model (Figure 10) this "phase locking" implies that the 3-s oscillation is colocated with the LLBL plasma and does not extend to the magnetosphere.

The 3-s oscillation does not have a preferred direction of magnetic field perturbation. To show this, we have plotted in Figure 13 the hodogram of the transverse magnetic field components after passing them through a high-pass filter with cutoff at $0.1 \mathrm{~Hz}$. Clearly, the perturbation has a random orientation. In terms of wave modes the 3-s oscillation in the boundary layer might be best described as an Alfvénic fluctuation, although we are unable to confirm the Alfvenic nature because of a lack of the measurements of electric field. However, Rezeau et al. [1989] combined magnetic and electric field measurements to support the Alfvenic nature of similar ULF waves observed by the GEOS 2 satellite near the magnetopause.

Retuming to Figures 7 and 8, we examine oscillations in the magnetosheath. The nature of field variations in the magnetosheath is highly variable, and we certainly do not claim that the particular segment shown is a typical example. Rather, it was chosen because the field was oriented northward and the spacecraft entered the magnetosphere within $5 \mathrm{~min}$ of the end of the illustrated time interval, at $7.1 R_{E}$, at $1030 \mathrm{MLT}$, and at $13^{\circ}$ dipole latitude. From this example we hope to find similarities or dissimilarities between oscillations just inside and outside the magnetopause under similar (northward) IMF orientations. 

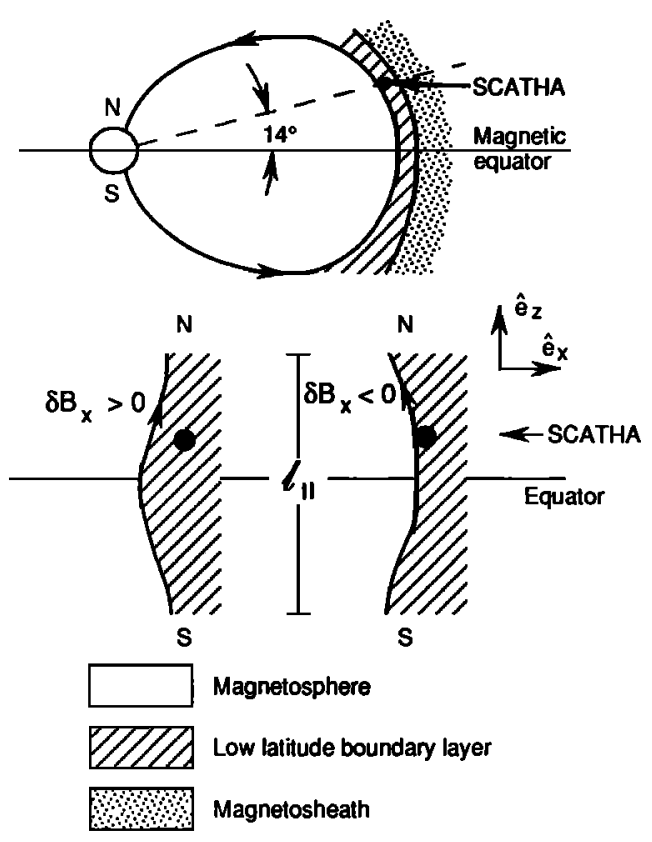

Fig. 10. (Top) A schematic for the satellite location with respect to the magnetic equator and the LLBL and (bottom) an enlanged view of the boundary layer at two epochs of $\delta B_{x}$

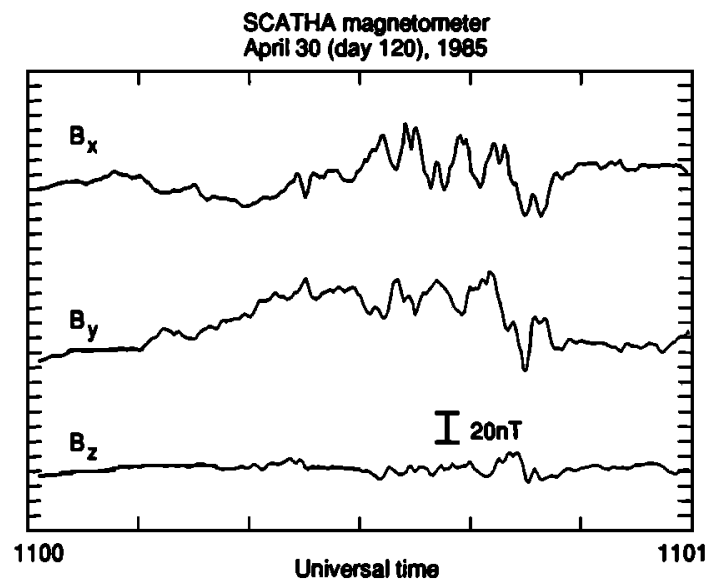

Fig. 11. High-time-resolution ( $0.25 \mathrm{~s})$ magnetic field data from SCATHA showing a 3-s oscillation in $B_{x}$ and $B_{y}$ superposed on a longer period (50 s) oscillation.
The magnetosheath spectra (Figure 8, lower panels) are characterized by $a f^{-2}$ background and by transverse oscillations between 0.1 and $1 \mathrm{~Hz}$, producing a peak near $0.6 \mathrm{~Hz}\left(-0.24 f_{c \mathrm{H}^{+}}\right)$. The polarization is left-handed. Similar waves with left-hand polarization have been observed in the magnetosheaths of the Earth below $f_{c \mathrm{H}^{+}}$[Schopke et al., 1990; B. J. Anderson, personal communication, 1990] and of Mercury above $f_{\mathrm{cH}^{+}}$[Fairfield and Behannon, 1976]. The latter authors identified the waves to be Doppler-shifted ion-cyclotron waves, and the same interpretation may apply to the SCATHA data, although we have observed frequencies substantially lower than the local proton gyrofrequency. At $f<0.1 \mathrm{~Hz}$ the transverse power is much weaker than in the boundary layer. This implies that the 50-s wave was generated within the boundary layer.

\subsection{Multisatellite Observations in the Magnetosphere}

In this section we study ULF waves in the magnetosphere, after briefly describing magnetospheric conditions during the compression of the magnetosphere.

Magnetic field and particle overview. Figure 14 shows a comparison of 3-s averaged magnetic field observations from the four spacecraft. The magnetic fields for GOES 5, GOES 6, and CCE are presented in the dipole VDH coordinates, where $\hat{e}_{H}$ (northward) is antiparallel to the Earth's dipole axis, $\hat{e}_{D}$ is magnetically east, and $\hat{e}_{V}=\ell_{D} \times \ell_{\psi}$ At 0923 UT the magnetic field was compressed at all spacecraft with $\Delta B_{T}$ ranging from $10 \mathrm{nT}$ at GOES 6 to $30 \mathrm{nT}$ at CCE. At GOES 6 and CCE, located at earlier local times, the field change was followed by $\sim 30 \mathrm{~min}$ of azimuthal field deflection. At each spacecraft, magnetic pulsations are seen after the compression. Then, starting at $\sim 1135$ UT, $B_{T}$ increased, probably as a result of an increase in solar wind dynamic pressure and at 1140 UT SCATHA was out in the magnetosheath (see also Figure 6 for the SCATHA data). By the time SCATHA entered the magnetosheath the sheath magnetic field had tumed strongly southward. The time of the southward tuming cannot be determined precisely, but it is likely that the solar wind dynamic pressure increase starting at 1135 UT accompanied a change in IMF orientation. After 1140 UT, southward magnetic field was observed by SCATHA until 1320 UT whenever the spacecraft was in the magnetosheath. The field direction is consistent with low-altitude observations. The two DMSP F7 polar cap passes at 1200 and 1305 UT (data not shown) strongly suggest a southward IMF configuration. There are two strong indicators. The first is a polar cap which

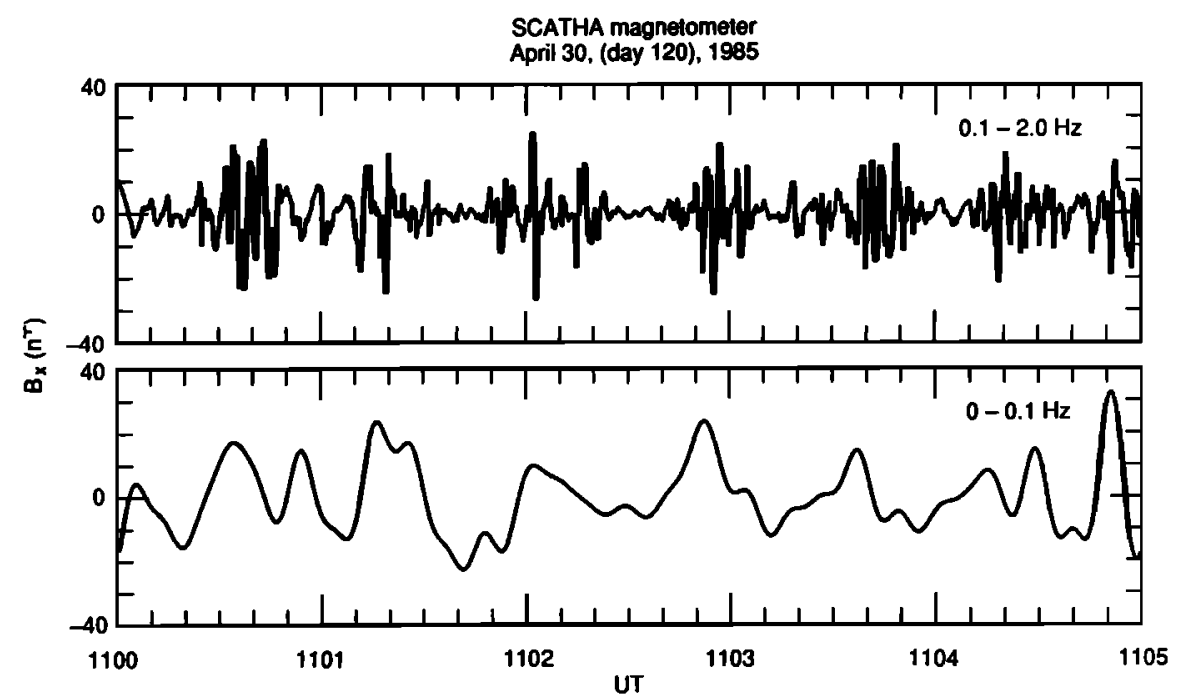

Fig. 12. High- and low-pass-filtered data showing an ervelope structure of higher-frequency oscillations and its relationship to the phase of a lower-frequency oscillation. 


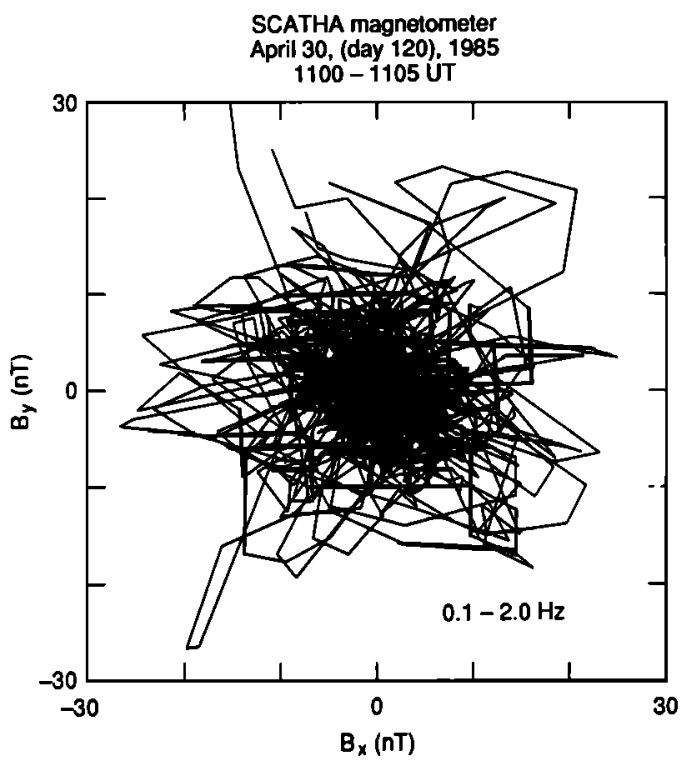

Fig. 13. A magnetic field hodogram for the higher-frequency oscillations in the boundary layer.
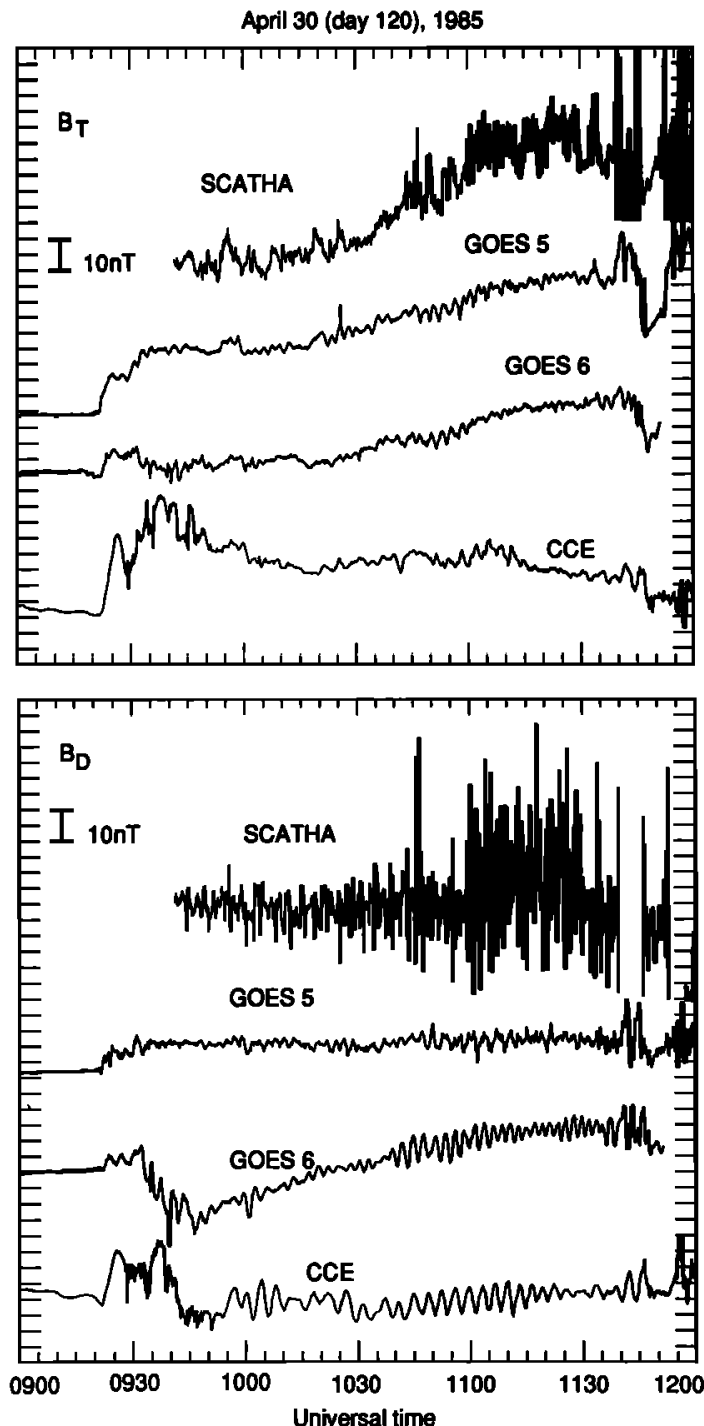

Fig. 14. Comparison of magnetic fields at the four satellites for the 0900-1200 UT interval. The data are all 3-s averages. is either void of precipitation or shows a clear polar rain indications (in this case the presence of a polar rain in the northern but not southern hemisphere suggests an away sector structure). The second indicator is that during an interval of southward IMF the dayside auroral oval tends to become smaller [e.g., Newell and Meng, 1987] and the nightside langer. Both these indicators imply that the IMF was southward during these passes.

Figure 15 shows ion data from MEPA on board CCE for a 6-hour interval encompassing the period of the magnetospheric compression. The trace in the upper frame is the dusk/dawn ratio of MCP1 counts at sectors covering $90^{\circ}$ pitch angle. The traces in the lower frame are ion fluxes averaged over the $32 \mathrm{MEPA}$ sectors and over four satellite spins ( $24 \mathrm{~s})$. There are three major ion flux increases in the interval shown: 0925, 1135, and 1242 UT. The first one is clearly associated with the SSC signature seen on the ground (Figure 2) and in the magnetosphere (Figure 14). The second one nearly coincides with the entry of SCATHA into the magnetosheath and with the magnetic field changes seen at the magnetospheric satellites. This dispersionless flux increase is probably due to a change in magnetospheric convection that was caused by southward turning of the IMF that probably accompanied the brief solar wind pressure increase seen by GOES 5 at 1140 UT (Figure 14). The third one is probably a substom effect.

The intensity of ion fluxes from 1000 to 1130 UT is rather steady. It does not show any evidence of ion injections caused by substorms. The absence of substorms was confirmed from inspection of Los Alamos energetic particle data from two geostationary satellites located near 1500 and 0200 MLT (D. N. Baker, personal communication, 1989). This result is consistent with our earlier inference from low-altitude precipitating particle data that the IMF was northward for the 1.5 -hour interval.

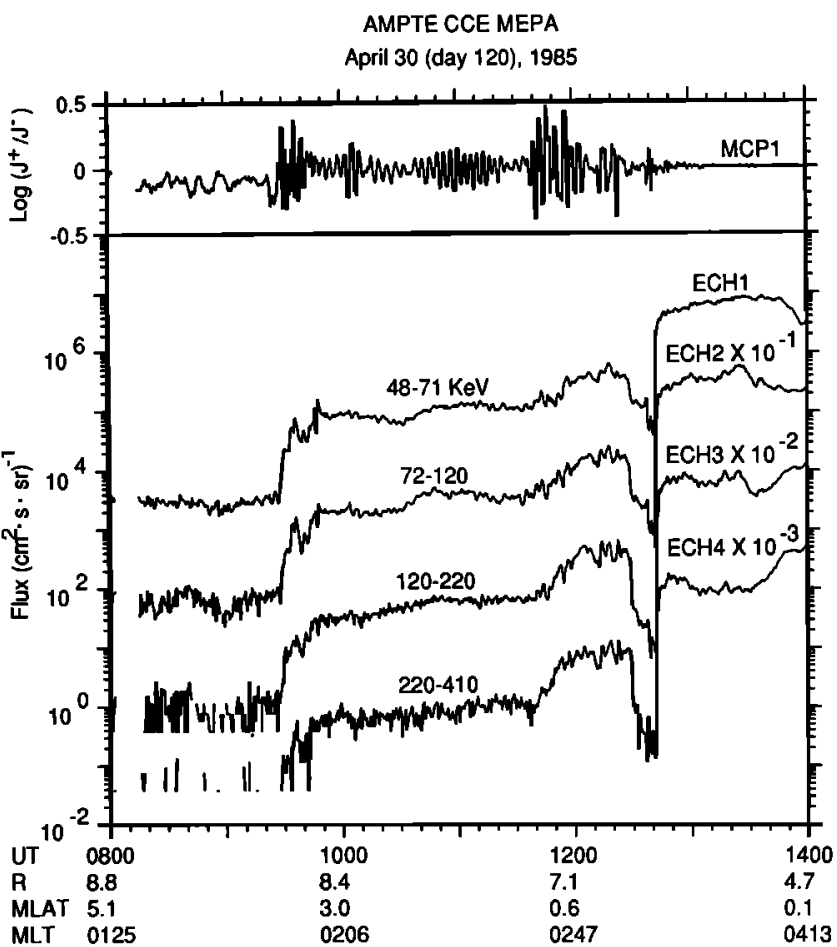

Fig. 15. Ion data from the MEPA instrument on CCE for the day 120 event. In the upper panel is the ratio (referred to as anisotropy in the text) of MCPl counts of the dawnward moving ions to those of the duskward moving ions at $90^{\circ}$ pitch angle. The lower panel shows 24-s (four spins) averages of ion fluxes measured by the ion head of MEPA. 
During this interval, as well as 30 - and 60 -min intervals before and after it, there was a clear oscillation in the ion dusk/dawn anisotropy with a period of $\sim 200 \mathrm{~s}$. The pulsation event was first reported by Lopez et al. [1986]. Inspection of particle data plotted in the same format as in Figure 15 for over 2-years of MEPA data has led us to realize that the day 120 event had an unusually large amplitude for an event occurring in the region from 0200 to 0300 MLT. This impression is substanciated by a recent statistical study by Anderson et al. [1990a]. They showed that magnetic Pc 5 toroidal-mode resonances at magnetic latitudes less than $13^{\circ}$ and near $L=8$ have a maximum occurrence rate of $40 \%$ at $0600 \mathrm{MLT}$ and that the rate decreases to $\sim 5 \%$ at $0200-0300 \mathrm{MLT}$.

Properties of magnetic pulsations. In this subsection we describe the properties of the magnetic pulsations. First, we show evidence that the pulsation at $\mathrm{CCE}$ is due to a fundamental toroidal oscillation [Radoski and Carovillano, 1966] of the local field line. This oscillation is characterized by a predominantly azimuthal magnetic field perturbation, and its frequency is approximately given by $f=\left[\delta d s / V_{A}\right]^{-1}$ where the integral is taken along a field line. Figure 16 shows a 1 -hour segment of ion flux anisotropy together with a component, $B_{E}$, of the magnetic field. The component $\hat{e}_{E}$ is parallel to $\langle B\rangle \times \hat{e}_{1}, \hat{e}_{a}$ being the spacecraft spin axis. The two quantities oscillate highly coherently. The cross spectral analysis shown in Figure 17 confirms that they oscillate with a phase lag of $90^{\circ}-100^{\circ}$. Because the particle anisotropy oscillation arises from the oscillatory $E \times B$ convection of the plasma [Lopez et al., 1986; Takahashi et al., 1988], the observed phase relation implies that the time-averaged Poynting flux along the ambient field is very small, a property of a standing wave. A similar technique was used to demonstrate the standing wave nature of a Pc 4 pulsation at geostationary orbit [Cummings et al., 1978].

The position dependence of pulsation properties can be seen clearly in the time plots of Figure 18 and the corresponding power spectra shown in Figure 19. These figures highlight the low-frequency end $(f<40 \mathrm{mHz}$, or period $>25 \mathrm{~s}$ ) of field variations that were not readily resolved in Plate 1 or Figure 8. The spectral properties are illustrated in terms of the compressional $\left(P_{2 z}\right)$ and transverse $\left(\boldsymbol{P}_{x x}+\boldsymbol{P}_{y y}\right)$ power densities, the perpendicular polarization parameters including the direction, $\phi$, of the major axis of polarization, percent polarization, and ellipticity, $\epsilon$. The angle $\phi$ is measured from the $+x$ axis toward the $+y$ axis, $\epsilon=1(-1)$ for righthanded (left-handed) circular polarization about the mean field, and $\epsilon=0$ for linear polarization.

Keeping in mind the spacecraft trajectories shown in Figure 4, we make the following observations. In the boundary layer

AMPTE CCE

April 30 (day 120), 1985

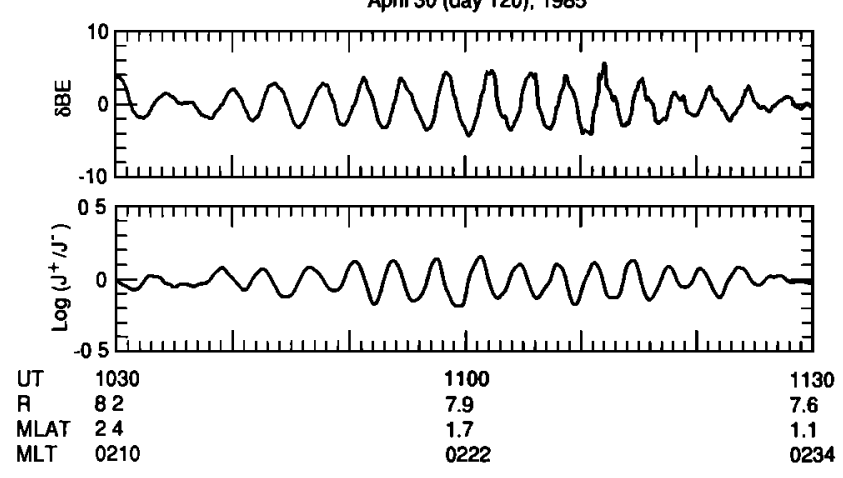

Fig. 16. Comparison of the (bottom) ion flux anisotropy oscillation from Figure 15 and the (top) aximuthal magnetic field for the same time interval.
AMPTE CCE

April 30 (day 120), 1985, $1030-1130$ UT

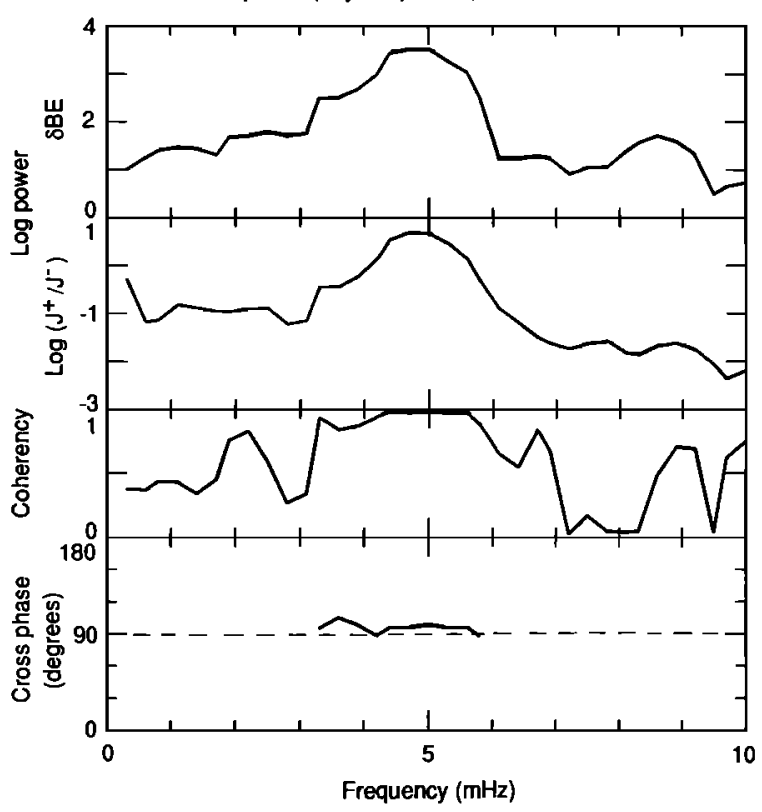

Fig. 17. Cross spectral analysis of the particle flux and magnetic field pulsations shown in Figure 16. The cross phase is shown only for frequency components with high $(>0.8)$ coherency.

(SCATHA) the overall power level is much greater than in the magnetosphere (other satellites). The transverse power is greater than the compressional power in the frequency band $4-40 \mathrm{mHz}$ by more than an order of magnitude. In this band, both components show a rather flat spectrum, but there is a weak peak at $20 \mathrm{mHz}$ in the transverse component. This arises from the type of oscillation shown in Figure 9. At this peak frequency (vertical dashed line) the transverse component is highly polarized with a left-hand elliptical polarization $(\epsilon \sim-0.5)$ and the major axis of polarization is nearly aligned with the aximuth $\left(\phi \sim 70^{\circ}\right)$.

A few Earth radii into the magnetosphere (GOES 5), the difference between the transverse and compressional components is smaller and the power falls off with frequency faster than in the boundary layer. The strongest peak is found in the transverse component at $5 \mathrm{mHz}$, where the field oscillation is linearly polarized $(\epsilon \sim-0.1)$ with the major axis oriented in the aximuthal direction $\left(\phi \sim 90^{\circ}\right)$.

Further into the magnetosphere (GOES 6 and CCE), the power spectra are dominated by a transverse oscillation at $7 \mathrm{mHz}$ (GOES 6) or $5 \mathrm{mHz}$ (CCE). Except for the frequency and the relatively large compressional power associated with the transverse oscillation at CCE the two spacecraft see similar features: linear polarization and a nearly aximuthally oriented major axis of polarization ( $\phi$ $-80^{\circ}$ ) at the spectral peak. We conclude that the 5-mHz oscillation at $\mathrm{CCE}$ is due to a toroidal-mode standing Alfven wave. The same should apply to the 7-mHz oscillation at GOES 6. It is not surprising that the compressional component at $\mathrm{CCE}$ oscillates at the same frequency as the transverse component. When a standing Alfven wave is established as a result of wave coupling in an inhomogeneous plasma, both the transverse and compressional components can exhibit a singular behavior (i.e., large-amplitude oscillation) as has been shown, for example, by Kivelson and Southwoood [1986]. The value of $\phi$ that is slightly smaller than $90^{\circ}$ implies that the coupling is with a tailward propagating source wave [Chen and Hasegawa, 1974]. 
Multisatellite magnetic field data Aprll 30 (day 120), 1985
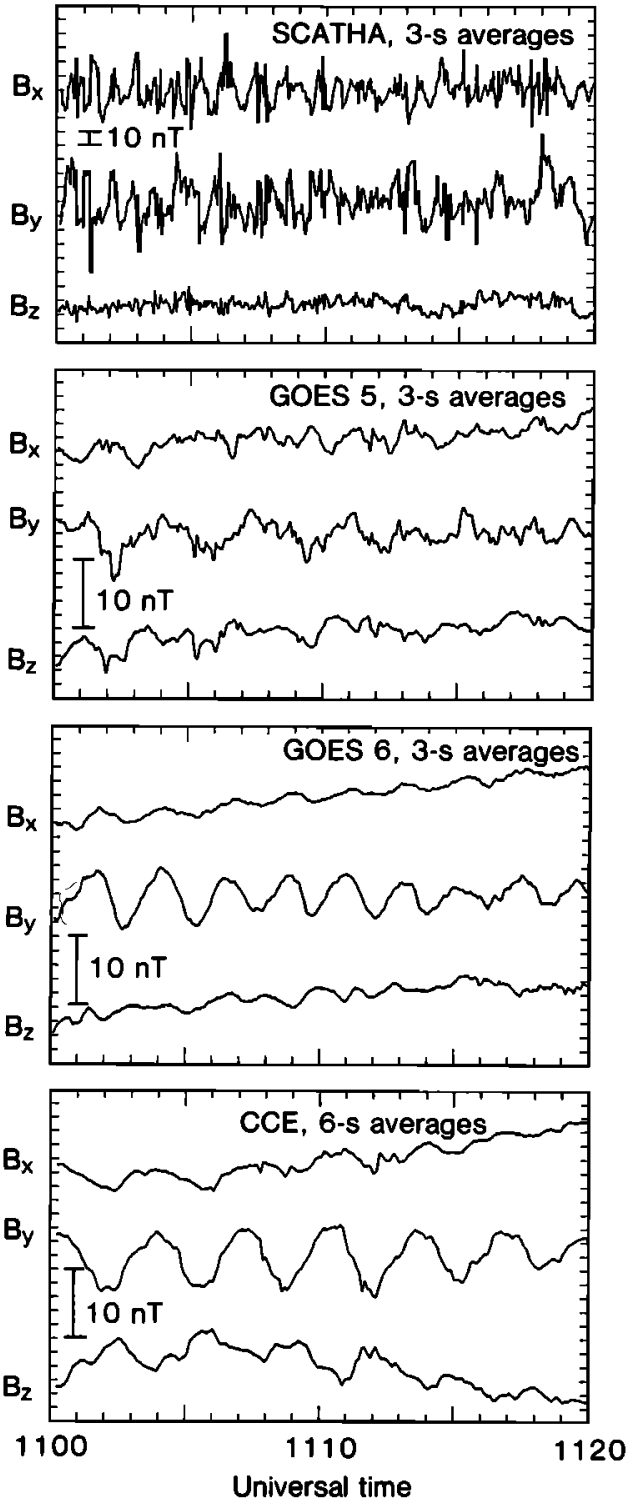

Fig. 18. Simultaneous observations of magnetic field oscillations in the boundary layer (SCATHA) and in the magnetosphere (GOES 5, GOES 6 , and $\mathrm{CCE}$ ). Field components are all given in the local mean-field-aligned coordinate described in the text.

\section{DISCUSSION}

In this section we discuss the excitation mechanisms of the observed ULF waves in the LLBL and magnetosphere.

\subsection{Origin of ULF Waves in the LLBL}

External pressure variations. Pressure variations in the solar wind or magnetosheath are an obvious candidate for the origin of ULF waves in the magnetosphere. The solar wind carries various plasma discontimuities and many of them accompany a pressure change. Even without such discontimuities in the solar wind, the bow shock can generate ULF waves. It has been recently suggested [Sibeck et al., 1989a] and demonstrated [Fairfield et al., 1990] that strong pressure pulses can be generated near the bow shock, get convected to the magnetopause, and give rise to transient magnetic field oscillations in the magnetosphere. The magnetospheric re- sponse to the pressure pulses is analogous to that for SC (sudden commencement) or SI (sudden impulse) [Sibeck et al., 1989b], but their repetition time scale ( $\sim 8 \mathrm{~min}$ for the Sibeck et al. events) as well as their possible small spatial scale makes them a different phenomenon. For these pulses, localized ringing of the magnetosphere may result [Farrugia et al., 1989; Southwood and Kivelson, 1990]. The irregular 5-10 min oscillation in $B_{T}$ seen both in the magnetosphere and in the LLBL can be attributed to such pressure pulses. However, it is questionable whether the same mechanism can be applied to the 50-s oscillations in the LLBL. Evidence against the pressure pulse mechanism are the periods which are shorter than those of typical pressure pulses and the strongly transverse magnetic field perturbations. Extemal pressure variations would cause compressional field variations.

We next discuss whether a 50-s wave could be generated in the upstream region, propagate to the magnetopause, and cause the observed 50-s oscillation. Upstream waves (periods near $30 \mathrm{~s}$ ) are a well-established phenamenon attributed to plasma instabilities occurring near the bow shock, and the relationship between the occurrences of the upstream waves and Pc 3-4 magnetic pulsations is also well established [Nourry, 1976]. A theoretical model by Takahashi et al. [1984b], for example, predicts an upstream wave frequency $f(\mathrm{mHz})=7.6 B(\mathrm{nT}) \cos ^{2} \theta_{X B}$, where $B$ is the magnitude of IMF in the upstream region and $\theta_{X B}$ is the angle between the Sun-

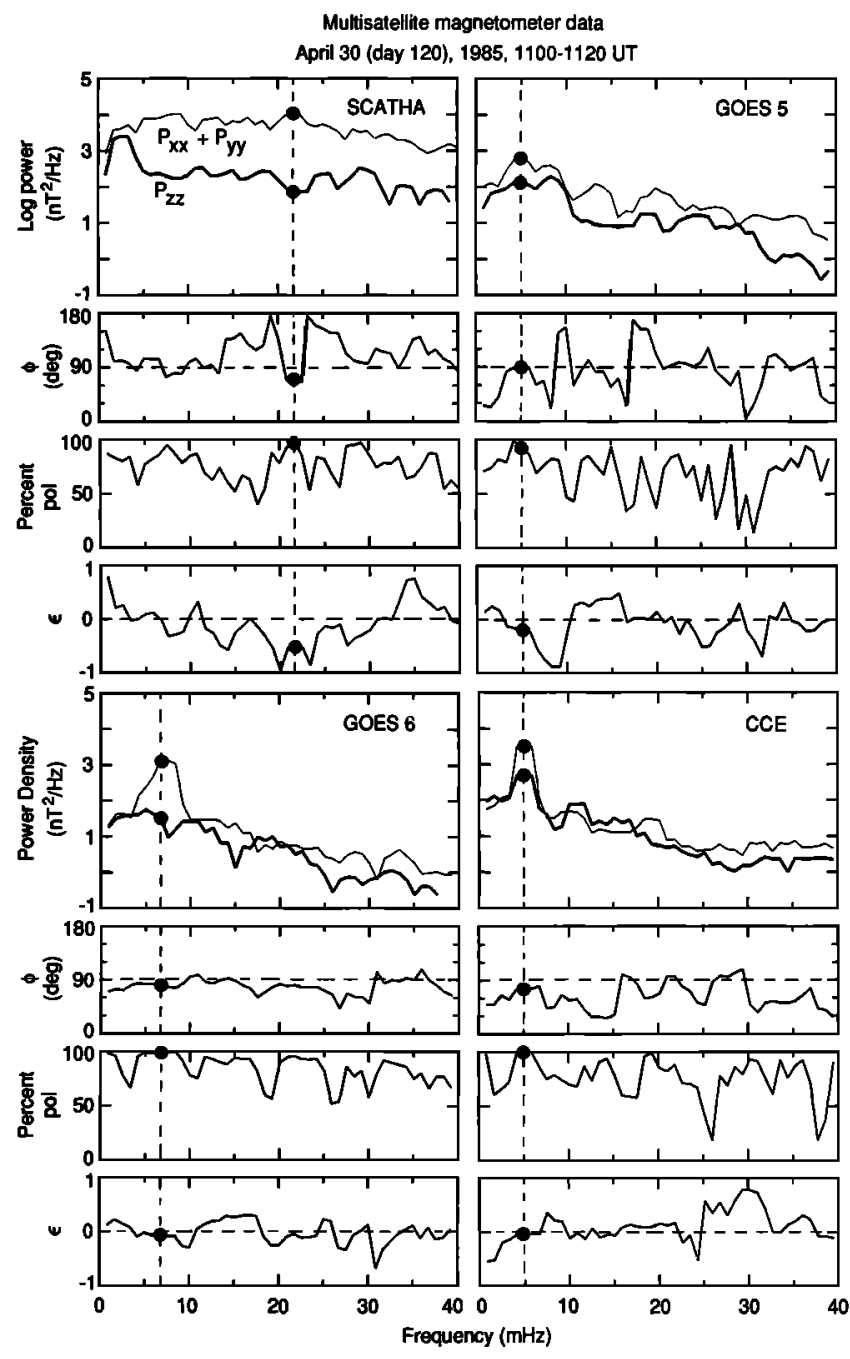

Fig 19. Power spectra of the magnetic field data shown in Figure 18. 
Earth line and the IMF. For a typical IMF cone angle $\theta_{x B}=45^{\circ}$ the formula implies that a 50-s wave corresponds to $B=5.3 \mathrm{nT}$. Such an IMF magnitude can easily occur. However, the observed polarization of the 50-s wave in the LLBL does not favor the transmission of the upstream wave into the magnetosphere. In order to propagate into the LLBL through the magnetopause the upstream wave should have a strong compressional component [Wolfe and Kaufmann, 1975] and the transmitted wave also should be strongly compressional. This transmission mechanism is certainly inconsistent with the strongly transverse nature of the 50-s wave. We conclude that the transmission of bow-shock-associated upstream waves is an unlikely mechanism for explaining the 50-s oscillation in the LLBL.

Kelvin-Helmholtz instability. We next discuss whether the 50-s wave was excited in the LLBL by a K.-H.-type instability. In the theory of the instability the magnetic field and plasma configuration for the equilibrium boundary is important. In particular, the strength of the velocity shear, the thickness of the boundary, and the magnetic field on the two sides of the boundary all contribute to the instability threshold and to the properties of waves excited [Southwood, 1968; Ong and Roderick, 1972; Lee et al., 1981; Walker 1981; Miura and Pritchett, 1982]. The ionospheric boundary conditions may also play an important role [Chen and Hasegawa, 1974].

In a plasma with a sheared flow, stability is provided by the magnetic field tension. If the fields on the two sides are parallel, they provide no restoring force to boundary distortions propagating perpendicular to the fields. Thus the boundary is unstable even with a very small velocity shear. We have inferred that IMF was northward at 0930-1130 UT, and if this is correct, then the sheath field could have been more nearly parallel to the geomagnetic field and the magnetopause is more likely to have been Kelvin-Helmholtz unstable. On the other hand, magnetic fields across the inner edge of the LLBL are nearly parallel, so the instability will occur even with a very small velocity shear [Ogilvie and Fitzenreiter, 1989]. We proceed with the assumption that the instability occurred either on the magnetopause or in the LLBL.

Consistent with the K.-H. instability which should generate waves that decay with distance from the boundary, the power spectral density (PSD) of magnetic field perturbations at the 50 -s period is substantially lower, well inside of the magnetosphere than in the LLBL. To quantify this point, we have listed in Table 1 the PSDs from the four satellites for the time interval of 1100 to 1120 UT. The distance $|x|$ from the magnetopause to each satellite was estimated using the magnetopause shape illustrated in Figure 4. For instance, the relative PSDs at GOES 5 (0600 MLT, magnetic latitude $=11^{\circ}$ north), which was closest to SCATHA, indicate that the wave power was down by a factor of $\sim 16\left(B_{2}\right)$ to 100 (transverse components) over a radial distance of $\sim 3 R_{E}$ from the LLBL. This result is inconsistent with a wave propagating away from the magnetopause but is consistent with a surface wave in the LLBL.
The frequency of a K.-H. wave may be determined in one of the following two ways. First, if the magnetospheric field lines are fixed at the ionosphere, then only a set of discrete parallel wave numbers $k^{(n)}$ are allowed, where $n$ is the harmonic number. According to Chen and Hasegawa [1974], who combined this idea with the MHD K.-H. instability threshold, the lowest (i.e., the fundamental) frequency $\omega$ and the perpendicular wave number, $k_{\perp}$, of the surface waves is given by $\omega=k_{\perp} \Delta V=k_{i l}^{(1)} V_{A}$, where $\Delta V$ is the jump in velocity across the shear, $V_{A}$ is the Alfven velocity, $k_{1}^{(n)}$ $\equiv n \pi / l$ with $n=1,2, \cdots, 3$, and $l$ is the length of the field line. In this case the frequency is first determined by the Alfven resonance condition and then $k_{\perp}$ follows. The observed 50 -s period is not unreasonable from this model. For a quantitative estimate we use a toroidal-mode equation [Cummings et al., 1969] assuming dipole field geometry but with twice the field strength to simulate the magnetic field near the magnetopause and a plasma mass density of $\sim 2 \mathrm{amu} / \mathrm{cm}^{3}$ calculated from the SCATHA ion measurements over the $0.074-15.6 \mathrm{keV}$ energy range in the LLBL (1100-1130 UT). The calculated period for the fundamental mode is $70 \mathrm{~s}$, which is not far from the observed period of $50 \mathrm{~s}$.

Second, if the velocity shear exists over a finite thickness $d$, then the most unstable wave will have a perpendicular wave number given by $k_{\perp} d \sim 0.5$ [Walker, 1981; Miura and Pritchett, 1982], and the corresponding frequency is given by $\omega \sim k_{\perp} \Delta V$. In this case the perpendicular wave number is determined first and then the frequency follows. This mechanism can again produce a 50 -s oscillation. For example, $\Delta V \sim 500 \mathrm{~km} / \mathrm{s}$ and $d \sim 1000 \mathrm{~km}$ give the observed frequency.

Theory also predicts the polarization of $\mathbf{K}$.-H waves. From tangential discontinuity models, one expects left-hand polarization on the magnetospheric side in the prenoon local time [Atkinson and Watanabe, 1966; Southwood, 1968; Dungey and Southwood, 1970]. If the boundary layer is included, the spatial pattern of polarization may become complicated. For example, in the Lee et al. [1981] model applied to the prenoon local time the "inner mode" will have a right-hand polarization between the magnetopause and the inner edge of the boundary layer, whereas the polarization will be left-handed inward of the inner edge of the boundary layer. In the same model the "magnetopause mode" will have a polarization pattern similar to that of the tangential discontinuity model. The observed left-handed polarization thus favors a magnetopause mode. However, since we are unable to determine the amplitude variation of the 50-s wave as a function of distance within the LLBL, it is not possible to conclude whether the 50-s wave really corresponds to the magnetopause mode.

\subsection{Excitation Mechanisms of Magnetospheric Pulsations}

Coupling of 50-s K.-H. wave to Alfvén wave. For a decade the prevailing idea for exciting magnetospheric pulsations was the field line resonance mechanism with a monochromatic source wave that is presumably excited by the $\mathrm{K}$.-H. instability at the

TABLE 1. Power Spectral Density at 50-s Period for 1100-1120 UT

\begin{tabular}{|c|c|c|c|c|c|c|c|}
\hline \multirow[b]{2}{*}{ Spacecraft } & \multirow{2}{*}{$\begin{array}{l}\mathbf{l x t}, \\
R_{E}\end{array}$} & \multicolumn{3}{|c|}{$\begin{array}{l}\text { Power Spectral } \\
\text { Density, } \mathbf{n T}^{2} / \mathbf{H z}\end{array}$} & \multicolumn{3}{|c|}{$\begin{array}{l}\text { Power Spectral Density } \\
\text { Relative to SCATHA }\end{array}$} \\
\hline & & $B_{x}$ & $B_{y}$ & $B_{z}$ & $B_{x}$ & $B_{y}$ & $B_{z}$ \\
\hline $\begin{array}{l}\text { SCATHA } \\
\text { GOES } 5 \\
\text { GOES } 6 \\
\text { CCE } 6\end{array}$ & $\begin{array}{l}<1 \\
\sim 3 \\
\sim 7 \\
-9\end{array}$ & $\begin{array}{c}3.1 \times 10^{3} \\
23 \\
0.87 \\
2.2\end{array}$ & $\begin{array}{c}4.5 \times 10^{3} \\
43 \\
5.8 \\
14\end{array}$ & $\begin{array}{c}1.7 \times 10^{2} \\
10 \\
8.3 \\
9.8\end{array}$ & $\begin{array}{c}1 \\
7.3 \times 10^{-3} \\
2.8 \times 10^{-4} \\
7.1 \times 10^{-4}\end{array}$ & $\begin{array}{c}1 \\
9.6 \times 1 \sigma^{-3} \\
1.3 \times 10^{-3} \\
3.1 \times 1 \sigma^{3}\end{array}$ & $\begin{array}{c}1 \\
5.9 \times 10^{-2} \\
4.9 \times 10^{-2} \\
5.8 \times 10^{-2}\end{array}$ \\
\hline
\end{tabular}


magnetopause [Chen and Hasegawa, 1974; Southwood, 1974; Walker et al., 1979]. In this theory the source wave was assumed to have the nature of a surface wave with the amplitude exponentially decaying with distance from the magnetopause. We examine this possibility. It is clear that the observed surface wave seen at SCATHA cannot couple with the simultaneously present magnetospheric pulsations at the mean period of $50 \mathrm{~s}$, because the period of the magnetospheric pulsation (200 s at GOES 5 and CCE and $150 \mathrm{~s}$ at GOES 6) was much longer than $50 \mathrm{~s}$.

Note, however, that the 50-s wave had a very broad spectrum $(\Delta f f \sim 1)$ and was accompanied by a compressional component which was much smaller than the transverse component in the LLBL but large compared to observations in the magnetosphere, as can be seen in Figure 18 and Figure 19. The power spectral density of the compressional component was $\sim 10^{2} \mathrm{nT}^{2} / \mathrm{Hz}$ over the band of 5 to $30 \mathrm{mHz}$ (30- to 200 -s period), which is higher than at any magnetospheric spacecraft except near $5 \mathrm{mHz}$ at CCE. Thus it is possible that broadband wave in the LLBL wave penetrated into the magnetosphere owing to its compressional component and coupled with standing shear Alfven waves at various locations and at various frequencies. A similar interpretation of ISEE spacecraft observations has been presented by Mitchell et al. [1990]. A theoretical basis for the broadband field line resonance has been presented by Hawsegawa et al. [1983].

It is important to note that higher-frequency components of the K.-H. waves decay faster with distance from the magnetopause, because those components have shorter wavelengths tangential to the magnetopause. Thus even if the spectrum of the K.-H. wave has a constant intensity over a band in the generating region, only lower-frequency components can penetrate deep into the magneosphere and are effective in resonantly exciting standing Alfvén waves. This gives a qualitative explanation as to why only the fundamental mode was strongly excited at CCE and GOES 6, while there is an indication of higher hamonic toroidal oscillations at GOES 5 (see the transverse spectrum in Figure 19, which exhibits a peak at 14 and $20 \mathrm{mHz}$ ).

Excitation of Alfuen waves by external pressure pulses. The field magnitude in the magnetosphere and in the LLBL exhibits irregular oscillations, and they may drive magnetospheric pulsations. In Figure 14 we pointed out that $B_{T}$ at SCATHA shows a quasi-periodic oscillation with peak separations of 5-10 $\mathrm{min}$ and peak-to-peak amplitude reaching $\sim 20 \mathrm{nT}$. The oscillation is present both in the magnetosphere (before 1035 UT at SCATHA) and in the LLBL (1035-1140 UT). Some of the major peaks in this oscillation are detected at GOES 5, for example at $\sim 0957, \sim 1020$, and 1034 UT, but the amplitude at GOES 5 is about half that at SCATHA. Therefore the 5-10 $\mathrm{min}$ oscillation appears to arise from solar wind pressure perturbations penetrating deep into the magnetosphere because of its presumably large azimuthal scale. Although the pulses are observed at 5-10 min intervals, it does not mean that they cannot excite pulsations at periods outside of 5-10 $\mathrm{min}$. In fact, each pulse can be considered to represent an impulsive source whose frequency spectrum is very broad. Thus the pulse train would in effect represent a broad-band quasi-steady source wave for exciting standing Alfven waves at position dependent frequency [e.g., Hasegawa et al., 1983].

Conversion of global cavity mode to Alfvén waves. More recently the consequences of impulsive sources and nonevanescent fast-mode disturbances have been discussed in the context of fastmode/Alfvén-mode coupling [Kivelson and Southwood, 1986; Allan et al., 1986; Inhester, 1987; Lee and Lysak, 1989]. These works show that the magnetosphere may resonate in a global mode in response to an impulsive extemal source with a large azimuthal scale ( $|m|<10$, where $m$ is the azimuthal wave number) and that the energy of the global mode may be transferred to the shear Alfvén wave in the same manner as in the original field line resonance mechanism. The global mode theory of Alfven wave excitation predicts that the global mode has a frequency that is constant everywhere in the magnetosphere and that the Alfvén wave is excited at the same frequency but on an $L$ shell on which the Alfven frequency matches the global mode frequency.

We do not consider that this scenario is applicable to the pulsations observed at GOES 5, GOES 6, and CCE at 1100 UT. The reason is that there was no great impulsive change in the field magnitude to drive the cavity mode and that there was no clear indication of a compressional oscillation with a single frequency over $L$ shells. It is even doubtful if a global cavity mode was excited at the sudden $B_{T}$ increase at 0923 UT. In Figure 14 we find that at each spacecraft a few cycles of oscillation follows the sudden compression. However, the period of the oscillation is again position dependent: $\sim 140 \mathrm{~s}$ at GOES $5, \sim 170 \mathrm{~s}$ at GOES 6, and $\sim 370 \mathrm{~s}$ at $\mathrm{CCE}$. These position-dependent periods are consistent with individual oscillation of $L$ shells. Such oscillations do not require the presence of a cavity mode [Radoski, 1974; Southwood, 1975; Poulter and Nielsen, 1982].

Effect of magnetospheric compression on pulsation amplitude. Regardless of the specific source mechanism, the CCE observation of large-amplitude Pc 5 pulsations appear to indicate, in addition to possible enhancement in source energy itself, the effect of the approach of the source region (magnetopause) to the spacecraft. The observed amplitude of a surface wave will depend on the distanc $|x|$ from the surface and the azimuthal wave length $\lambda$ of the wave as $\exp (-2 \pi \mid x / \lambda)$ [Lanzerotti et al., 1981], and the locally excited Alfven waves by coupling with the surface wave should exhibit a similar amplitude dependence on $|x|$ and $\lambda$ [e.g., Southwood, 1975]. If we assume, for example, $\lambda=10 R_{E}$, then this expression implies that, at the CCE location $\left(|x| \sim 9 R_{E}\right)$ shown in Figure 4 , the wave amplitude would be about 20 times larger when the subsolar magnetopause was located at $7 \boldsymbol{R}_{E}$ than at the nominal $11 R_{E}$ (bxt $\sim 14 R_{E}$ ). This enormous amplification factor is certainly sufficient to account for the unusually large amplitude of the Pc 5 wave observed at CCE. Furthermore, because a high solar wind velocity would mean a smaller magnetosphere, we speculate that this geometric effect may account for a good portion of the positive correlation between the amplitude of magnetospheric Pc 3-5 pulsations and the solar wind velocity reported previousiy [Greenstadt et al., 1979; Wolfe, 1980].

\section{Conclusions}

In conclusion we have analyzed combined data from five spacecraft to understand the structure of the LLBL, the ULF waves in the LLBL, and the relation of the LLBL waves to magnetic pulsations in the magnetosphere. Both the intensity and the pitch angle distribution of the particles clearly show the presence of the LLBL for the time interval studied. The average magnetic field is rotated slightly at or near the LLBL/magnetosphere interface, which is consistent with a field-aligned current sheet with the region 1 flow direction. Our major results concerning ULF waves are summarized as the following:

1. A 5-10 min compressional perturbation is present both in the LLBL and the magnetosphere. This is most likely caused by pressure perturbations in the solar wind, because the perturbation was observed well inside the magnetosphere.

2. In the boundary layer, large-amplitude transverse oscillations are present. These can be separated into a 50-s oscillation and a higher frequency oscillation. The 50-s is likely a surface wave 
excited by the K.-H. instability because the amplitude of the wave diminished quickly with distance from the magnetopause. The origin of the higher-frequency oscillation is unknown.

3. Magnetic Pc 4-5 pulsations are present in the magnetosphere with aximuthal perturbations and position-dependent frequency. They can be explained by toroidal mode standing Alfven waves.

4. The magnetic pulsations can be attributed to a broadband energy source covering the Pc 4-5 band. The 5-10 min quasiperiodic pressure pulses or a broadband compressional background in the LLBL may correspond to the broadband source. We do not see any need to invoke the global cavity mode to explain the pulsations.

Acknowledgments. We are grateful to J. F. Fennell and D. R. Croley of the Aerospace Corporation for generating SCATHA magnetic field and particle data files and also for various comments on the interpretation of the data. We thank T. A. Potemra and L. J. Zanetti for the use of AMPTE CCE magnetic field data and R. W. McEntire for the use of AMPTE CCE medium-energy particle data. Thanks are also due to B. J. Anderson, R. E. Lopez, H. Luehr, and P. Song for helpful comments. The GOES 5 and GOES 6 magnetic field data were made available by the National Geophysical Data Center, Boulder, Colorado. The Air Force DMSP particle data set was obtained from the World Data Center A in Boulder Colorado; D. Hardy and colleagues designed and built the instrument. The $A E$ index was provided by the Data Analysis Center for Geomagnetism and Spacemagnetism, Kyoto University. Work at The Johns Hoplins University Applied Physics Laboratory was supported by NASA mder Task I of Navy contract N00039-89-5301, by the Atmospheric Sciences Division, National Science Foundation under grant ATM-8713212, and by the Air Force Office of Scientific Research under grant 88-0101. The work performed at The Aerospace Corporation was supported by the U.S. Air Force System Commands' Space System Division under contract F04701-88-C-0089.

The Editor thanks A. Wolfe and another referee for their assistance in evaluating this paper.

\section{REFERENCES}

Allan, W., S. P. White, and E. M. Poulter, Impulse-excited hydromagnetic cavity and field-line resonances in the magnetosphere, Planet. Space Sci., 34, 371, 1986.

Anderson, B. J., M. J. Engebretson, S. P. Rounds, L. J. Zametti, and T. A. Potemra, A statistical study of Pc 3-5 pulsations observed by the AMPTE/CCE magnetic field experiment, 1, Occurrence distribations, $J$. Geophys. Res., 95, 10,495, 1990a.

Anderson, B. J., K. Taloghashi, R. E. Erlandson, and L. J. Zametti, Pc 1 pulsations observed by AMPTE/CCE in the Earth's outer magnetosphere. Geophys. Res. Lett., 17, 1853, 19906.

Atrinson, G., and T. Watanabe, Surface waves on the magnetospheric boundary as possible origin of long period geomagnetic micropulsations, Earth Planet. Sci. Lett., 1, 89, 1966.

Aubry, M. P., M G. Kivelson, and C. T. Russell, Motion and structure of the magnetopause, J. Geophys. Res., 76, 1673, 1971.

Bryant, D. A., and S. Riggs, At the edge of the Earth's magnetosphere: A survey by AMPTE-UKS, Philos. Trans. R. Soc. London Ser., A 328, 43, 1989.

Burch, J. L., P. H. Reiff, and M. Sugiura, Upward electron beams measured by DE-1, A primary source of dayside region 1 Birkeland currents, Geophys. Res. Lett., 10, 753, 1983.

Chen, L., and A. Hasegawa, A theory of long-period magnetic pulsations, 1, Steady state excitation of field line resonance, J. Geophys. Res., 79, 1024, 1974.

Collin, H. L., R. D. Sharp, and E. D. Shelley, The occurrence and characteristics of electron beams over the polar regions, J. Geophys. Res., 87, 7504, 1982.

Couzens, D., G. K. Parks, K. A. Anderson, R. P. Lin, and H. Reme, ISEE particle observations of surface waves in the magnetopause boundary layer, J. Geophys. Res., 90, 6343, 1985.

Cummings, W. D., R. J. O' Sullivan, and P. J. Coleman, Jr., Standing Alfven waves in the magnetosphere, J. Geophys. Res., 74, 778, 1969.

Cummings, W. D., S. E. DeForest, and R. L. McPherron, Measurements of the Poynting vector of standing hydromagnetic waves at geosynchronous orbit, J. Geophys. Res., 83, 697, 1978.

Dungey, I. W., and D. J. Southwood, Ultra low frequency waves in the magnetosphere, Space Sci. Rev., 10, 672, 1970.
Engebretson, M. J., L. J. Zanetti, T. A. Potemra, and M. H. Acuna, Harmonically structured ULF pulsations observed by the AMPTE CCE magnetic field experiment, Geophys. Res. Lett., 13, 905, 1986.

Easman, T. E., E. W. Hones, Jr., S. J. Bame, and J. R. Asbridge, The magnetospheric boundary layer: Site of plasma, momentum and energy transfer from the magnetosheath into the maghetosphere, Geophys. Res. Lett., 3, 685, 1976.

Fairfield, D. H., Average and unusual locations of the Earth's magnetopause and bow shock, J. Geophys. Res., 76, 6700, 1971.

Fairfield, D. H., and K. W. Behannon, Bow shock and magnetosheath waves at Mercury, J. Geophys. Res., 81, 3897, 1976.

Fairfield, D., W. Baumjohann, G. Paschmann, H. Luhr, and D. Sibeck, Upstream pressure variations associated with the bow shock and their effects on the magnetosphere, J. Geophys. Res., 95, 3773, 1990.

Farrugia, C. J., R. P. Riinbeek, M. A. Saunders, D. J. Southwood, D. J. Rodgers, M. F. Smith, C. P. Chaloner, D. S. Hall, P. J. Christiansen, and L. J. Woolliscroft, A multi-instrument study of flux transfer event structure, J. Geophys. Res., 93, 14,465, 1988.

Farrugia, C. J., M. P. Freeman, S. W. Cowley, D. J. Southwood, and M. Lockwood, Pressure-driven magnetopause motions and attendant response on the ground, Planet. Space Sci., 37, 589, 1989.

Fennell, J. F., Description of P78-2 (SCATHA) satellite and experiments, in The IMS Source Book, edited by C. T. Ruissell and D. J. Sonthwood, p. 65, AGU, Washington, D. C., 1982.

Greenstadt, E. W., H. J. Singer, C. T. Russell, and J. V. Olson, , MMF orientation, solar wind velocity, and Pc 3-4 signals: A joint distribution, J. Geophys. Res., 84, 527, 1979.

Greenwald, R. A., and A. D. M. Walker, Enengetics of long period resonant hydromagnetic waves, Geophys. Res. Lett., 7, 745, 1980.

Grubb, R. N., The SMS/GOES Space Environment Monitor Subsystem, Tech. Memo SEL-42, Nat1. Oceanic and Atmos. Admin., Environ. Res. Lab., Boulder, Colo., 1975.

Hardy, D. A., L. K. Schmitt, M. S. Gussenhoven, F. J. Marshall, H. C. Yeh, T. L. Shumaker, A. Hube, and J. Pantazis, Precipitating electron and ion intensity (SSJ/4) for the block 5D/flights 6-10 DMSP satellites: Calibration and data presentation, Rep. AFGL-TR-84-0317, Air Force Geophys. Lab., Hanscom Air Force Base, Mass., 1984.

Hardy, D. A., M. S. Gussenhoven, K. Riehl, R. Burkhardt, N. Heinemann, and T. Schmmaker, The characteristics of polar-cap precipitation and their dependence on the interplanetary magnetic field and the solar wind, in Solar Wind-Magnetosphere Coupling, edited by Y. Kamide and J. A. Slavin, p. 575, TERRAPUB, Tokyo, 1986.

Hasegawa, A., K. H. Tsui, and A. S. Assis, A theory of long period magnetic pulsations, 3, Local field line oscillations, Geophys. Res. Lett., 10, 765, 1983.

Hones, E. W., Jr., Magnetic structure of the boundary layer, Space Sci. Rev., 34, 201, 1983.

Iijima, T., and T. A. Potemra, Field-aligned currents in the dayside cusp observed by Triad, J. Geophys. Res., 81. 5971, 1976.

Inhester, B., Numerical modeling of hydromagnetic wave structure in the magnetosphere, J. Geophys. Res., 92, 4751, 1987.

Kivelson, M. G., and D. J. Southwood, Coupling of global magenetospheric MFD eigenmodes to field line resonances, J. Geophys. Res., 91, 4345, 1986.

Klumpar, D. M, and W. J. Heilkila, Electrons in the ionospheric source cone, evidence for nuaway electrons and carriers of downward Birkeland currents, Geophys. Res. Lett., 9, 873, 1982.

Lanzerotti, L. J., L. V. Medford, C. G. Maclennan T. Hasegawa, M. H. Acuna, and S. R. Dolce, Polarization characteristics of hydromagnetic waves at low geomagnetic latitudes, J. Geophys. Res., 86, 5500, 1981.

Lassen, K., C. Danielsen, and C.-I. Meng, Quiet-time average auróral configuration, Planet. Space Sci., 36, 791, 1988.

Ledley, B. G., Magnetopause attitades during Ogo 5 crossings, J. Geophys. Res., 76, 6736, 1971.

Lee, D.-H., and R. L. Lysak, Magnetospheric ULF wave coupling in the dipole model: The impulse excitation, J. Geophys. Res., 94, 17,097. 1989.

Lee, L. C., R. K. Albano, and J. R. Kan, Kelvin-Helmholtz instability in the magnetopause-boundary layer region, J. Geophys. Res., 86, 54, 1981.

Lopez, R. E., M. J. Engebretson, R. W. McEntire, A. T. Y. Lui, L. J. Zanetti, T. A. Potemra, and S. M. Krimigis, The response of energetic particles to nightside magnetic polsations as seen by AMPTE/CCE, Adv. Space Res., 6, 235, 1986.

McEntire, R. W., E. P. Keath, D. E. Fort, A. T. Y. Lui, and S. M. Krimigis, The medium-energy particle analyzer (MRPA) on the AMPTE/CCE spacecraft, IEEE Trans. Geosci. Remote Sens., GE-23, 230, 1985. 
Mead, G. D., Deformation of the geomagnetic field by the solar wind, $J$. Geophys. Res., 69, 1181, 1964.

Mitchell, D. G., M. J. Engebretson, D. J. Williams, C. A. Cattell, and R. Lundin, Pc 5 pulsation in the outer dawn magnetosphere seen by ISEE 1 and 2, J. Geophys. Res., 95, 967, 1990.

Miura, A., and P. L. Pritchett, Nonlocal stability analysis of the MFD Kelvin-Helmholtz instability in a compressible plasma, J. Geophys. Res., 87, 7431, 1982.

Newell, P. T., and C.-I. Meng, Cusp width and $B_{z}$ : Observations and a conceptual model, $J$. Geophys. Res., 92, 13,673, 1987.

Nourry, G. R, Interplanetary magnetic field, solar wind and geomagnetic pulsation, Ph.D. thesis, Univ. of British Columbia, Vancouver, 1976.

Ogilvie, K. W., and R. J. Fitzenreiter, The Kelvin-Helmholtz instability at the magnetopause and inner boundary layer surface, $J$. Geophys. Res., 94, 15,113, 1989.

Ogilvie, K. W., R. J. Fitzenreiter, and J. D. Scudder, Observations of electron beams in the low-latitude boundary layer, J. Geophys. Res., 89, 10,723, 1984.

Ong, R. S. B., and N. Roderick, On the Kelvin-Helmholtz instability of the Barth's magretopause, Planet. Space Sci., 20, 1, 1972.

Potemra, T. A., L. J.Zanetti, and M H. Acuna, The AMPTE/CCE magnetic field expetiments, IREE Trans. Geosci. Remote Sensing. GE-23, 246, 1985.

Potemra, T. A., L. J. Zanetti; P. F. Bythrow, R. E. Erlandson, R. Lundin, G. T. Marklund, L. P. Block, and P.-A. Lindqvist, Resonant geomagnetic field oscillations and Birkeknd currents in the morning sector, J. Geophys. Res., 93, 2661, 1988.

Poulter, E. M, and E. Nielsern, The hydromagnetic oscillation of individual shells of the ceomannetic field, J. Geophys. Res., 87, 10,432, 1982.

Radoski, H. R, A theory of latitude dependent geomagnetic micropulsations: The asymptotic fields, J. Geophys. Res., 79, 595, 1974.

Radoski, H. R., and R. L. Carovillano, Axisymmetric plasmasphere resonances, toroidal mode, Phys. Fluids, 9, 285, 1966.

Rezeac, L., A. Morane, S. Perraut, A. Roux, and R. Schmidt, Characterization of Alveric fluctuations in the magnetopanse boundary layer, $J$. Geophys. Res., 94, 101, 1989.

Sckopke, N., G. Paschmann, G. Haerendel, B. U. O. Sonnerup, S. J. Bame, T. G. Forbes, E. W. Hones, Jr., and C. T. Russell, Structure of the lowlatitude boundary layer, $J$. Geophys. Res., 86, 2099, 1981.

Sckopke, N., G. Paschimann, A. L. Brinca, C. W. Carlson, and H. Luehr, Ion thermalization in quasi-perpendicular shocks involving reflected ions, $J$. Geophys. Res., 95, 6337, 1990.

Sharp, R. D., E. G. Shelley, R. G. Johnson, and A. G. Ghielmetti, Counterstreaming electron beams at altitudes of $\sim 1 R_{E}$ over the auroral zone, $J$. Geophys. Res., 85, 92, 1980.

Sibeck, D. G., A model for the transient magnetospheric response to sudden solar wind dynamic pressure variations, J. Geophys. Res., 95, $3755,1990$.

Sibeck, D. G., W. Baumjohann, R. C. Elphic, D. H. Fairfield, J. F. Fennell, W. B. Gail, L. J. Lanzerotti, R. E. Lopez, H. Luehr, A. T. Y. Lui, C. G. Maclennan, R. W. McEntire, T. A. Potemra, T. J. Rosenberg, and $K$. Takahashi, The magnetospheric response to 8-minute period strong-amplitude upstream pressure variations, J. Geophys. Res., 94, 2505, 1989a.
Sibeck, D. G., W. Baumjohann, and R. E. Lopez, Solar wind dynamic pressure variations and transient magnetospheric signatures, Geophys. Res. Lett., 16, 13, 19896.

Sibeck, D. G., R. P. Lepping, and A. J. Lazarus, Magnetic field line draping in the plasmn depletion layer, $J$. Geophys. Res., 95, 2433, 1990.

Song, P., R. C. Elphic, C. T. Russell, J. T. Gosling, and C. A. Cattell, Structure and properties of the subsolar magnetopanse for northward IMF: ISEB observations, J. Geophys. Res., 95, 6375, 1990.

Southwood, D. J., The hydromagnetic stability of the magnetospheric boundary, Planet. Space Sci., 16, 587, 1968.

Southwood, D. J., Some features of field line resonances in the magnetosphere, Planet. Space Sci., 22, 1974.

Southwood, D. J., Comments on field line resonances and micropulsations, Geophys. J. R. Astron. Soc., 41, 425, 1975.

Southwood, D. J., and M. G. Kivelson, The magnetohydrodynamic response of the magnetospheric cavity to changes in solar wind pressure, $J$. Geophys. Res., 95, 2301, 1990.

Sugtura, M., and C. R. Wilson, Oscillation of the geomagnetic field lines and associated magnetic perturbations at conjugate points, J. Geophys. Res., 69, $1211,1964$.

Thkahashi, $K$., and R. L. McPherron, Harmonic structure of Pc 3-4 pulsations, J. Geophys. Res., 87, 1504, 1982.

Thkahashi, K. R. L. McPherron, and W. J. Hughes, Multisatellite observations of the harmonic structure of Pc 3-4 magnetic polsations, $J$. Geophys. Res., 89, 6758, 1984a.

Takahashi, K., R. L. McPherron, and T. Terasawa, Dependence of the spectrum of Pc 3-4 pulsations on the interplanetary magnetic field, $J$. Geophys. Res., 89, 2770, $1984 b$.

Takshashi, K., L. M. Kistler, T. A. Potemre, R. W. McEntire, and L. J. Zanetti, Magnetospheric ULF waves observed during the major magnetospheric compression of November 1, 1984, J. Geophys. Res., 93, 14,369, 1988.

Walker, A. D. M., The Kelvin-Helmholtz instability in the low-latitude boundary layer, Planet. Space Sci., 29, 1119, 1981.

Walker, A. D. M. R. A. Greenwald, W. F. Stuart, and C. A. Green, Stare auroral radar observations of Pc 5 geomagnetic pulsations, J. Geophys. Res., 84, 3373, 1979.

Wolfe, A., Dependence of mid-latitade hydromagnetic energy spectra on solar wind speed and interplanetary magnetic field direction, $J$. Geophys. Res., 85, 5977, 1980.

Wolfe, A., and R. L. Kanfmann, MHD wave transmission and production near the magnetopause, J. Geophys. Res., 80, 1764, 1975.

P. T. Newell, D. G. Sibeck, and K. Takahashi, The Johns Hopkins University, Applied Physics Laboratory, Laurel, MD 20732.

H. E. Spence, Space Sciences Laboratory, The Aerospace Corporation, B1 Segundo, CA 90245.
(Received October 18, 1990; revised January 25, 1991; accepted February 8, 1991.) 